\title{
Gauge links for transverse momentum dependent correlators at tree-level
}

\section{M.G.A. Buffing and P.J. Mulders}

Nikhef and Department of Physics and Astronomy, VU University Amsterdam, De Boelelaan 1081, NL-1081 HV Amsterdam, the Netherlands

E-mail: m.g.a.buffing@vu.nl, mulders@few.vu.nl

ABSTRACT: In this paper we discuss the incorporation of gauge links in hadronic matrix elements that describe the soft hadronic physics in high energy scattering processes. In this description the matrix elements appear in soft correlators and they contain non-local combinations of quark and gluon fields. In our description we go beyond the collinear approach in which case also the dependence on transverse momenta of partons is taken into consideration. The non-locality in the transverse direction leads to a complex gauge link structure for the full process, in which color is entangled, even at tree-level. We show that at tree-level in a 1-parton unintegrated (1PU) situation, in which only the transverse momentum of one of the initial state hadrons is relevant, one can get a factorized expression involving transverse momentum dependent (TMD) distribution functions. We point out problems at the level of two initial state hadrons, even for relatively simple processes such as Drell-Yan scattering.

Keywords: Parton Model, QCD, Jets

ArXiv EPRINT: 1105.4804 


\section{Contents}

1 Introduction 1

2 Collinear and Transverse Momentum Dependent (TMD) correlators 3

3 Color gauge invariance $\quad 8$

3.1 Collinear gluons 9

$\begin{array}{lll}3.2 & \text { Collinear correlators } & 12\end{array}$

$\begin{array}{ll}3.3 & \text { Including transverse gauge connections } \\ \end{array}$

4 Disentangling the color flow dependence $\quad \mathbf{1 5}$

$\begin{array}{lll}\text { 4.1 TMDs in 'elementary' processes } & 16\end{array}$

$\begin{array}{lll}\text { 4.2 TMDs in 1-parton unintegrated processes } & 17\end{array}$

5 Analysis in terms of transverse moments $\quad 19$

$\begin{array}{ll}5.1 \text { Single weighted asymmetries } & 19\end{array}$

5.2 Double weighted asymmetries 24

$\begin{array}{lll}6 & \text { Conclusions } & 27\end{array}$

$\begin{array}{ll}\text { A Collinear gauge links } & 30\end{array}$

B Intertwined gauge connections $\quad 32$

\section{Introduction}

In the description of high energy processes involving hadrons one aims at isolating the underlying hard process in terms of partons, quarks and gluons. The purpose of this can be twofold. The aim might be to study the quark and gluon structure of hadrons, or it might be to account for the soft hadronic physics to study unknown details in the hard process, for instance involving physics beyond the Standard Model. In both cases one identifies a number of soft functions among them distribution functions $f^{H \rightarrow i}(x)$ and fragmentation functions $D^{h \rightarrow i}(z)$ for quarks $(i=q$, where $q$ is an (anti-)quark flavor) and gluons $(i=g)$, which have a natural interpretation as the probability of finding a parton $i$ with momentum fractions $x$ in a hadron $H$ or as measure of the number of hadrons $h$ with momentum fractions $z$ in the 'decay' of a parton $i$. We will refine the definitions of these fractions below. To some level of accuracy, one can express observables such as cross sections and asymmetries in terms of these distribution and fragmentation functions. Going beyond the collinear treatment, one includes the dependence on transverse momenta. These momenta can serve as degrees of freedom in the connection between 
hadrons and partons very much like spin degrees of freedom. In the case of fragmentation, transverse momentum is in essence just the mismatch between parton momentum $k$ and hadron momentum $K_{h}$, or better between the fraction of the parton momentum $z k$ and $K_{h}$. This, at least intuitively, corresponds for jet fragmentation to identifying the parton momentum with an appropriately defined jet direction. Also for initial state hadrons one can include dependence on transverse momentum, which is the mismatch between an appropriate fraction of the hadron momentum $x P$ and the parton momentum $p$. In a high energy scattering process, one is able to use the presence of a hard scale to identify parton momenta (integration variables) with accessible combinations of external momenta. This is best known for the momentum fractions, but it is also possible for the transverse momenta.

In the present paper, we remain at tree-level (to be made more explicit), which implies that the intuitive language can in most cases also be used at the level of the matrix elements of quark and gluon fields that constitute correlators [1], which in turn are parametrized in terms of the beforementioned soft functions connecting partons and hadrons [2]. A complication that arises already at tree-level, is that the appropriate combinations of quark and gluon fields in the correlators need to be gauge-invariant combinations. This would trivially be the case for local products of fields, but already in the collinear treatment which studies the dependence on the momentum fractions $x$ and $z$, the partonic field combinations are non-local along a light-like direction conjugate to the parton momentum. Then one finds that gluon fields with polarizations along the momentum direction, which naturally appear in a twist analysis of leading operators, need to be resummed to make up the required path ordered exponentials or Wilson lines connecting the non-local parton fields [3]. Although this involves an infinite number of additional gluons, we still refer to this resummation as tree-level, since one resums leading combinations of coupling constant and field $(g A)$. Although their momentum is integrated over, these gluons don't appear in loops but as additional gluons connecting the soft and hard parts and as such are at the same level as other partons. The procedure is in fact ensuring that the non-locality is color gauge invariant, i.e. the replacement $i \partial_{\mu} \rightarrow i D_{\mu}=i \partial_{\mu}+g A_{\mu}$. The importance of the directions of gauge links was realized in ref. [4]. Even in the case of a light-like non-locality, the links are either pastor future-pointing, but in the absence of transverse separation this feature becomes irrelevant in the squared amplitude. In the case of transverse momentum dependent (TMD) correlators [5] and correspondingly TMD soft functions [6, 7], one also must account for a transverse non-locality, requiring more complicated Wilson lines. These Wilson lines have been extensively studied [8-13]. The TMDs lead to a rich phenomenology of azimuthal asymmetries $[6,7,14,15]$. The distinction of past- and future-pointing gauge links provides a natural explanation of single spin asymmetries at the partonic level. Through the gauge links, time-reversal odd (T-odd) parts are incorporated in the TMD correlators and the soft functions in their parametrization within a field theoretical framework of Quantum Chromodynamics (QCD). Beyond tree-level, many complications arise [16-18], of which at present certainly not all implications have been investigated. Although higher orders in QCD may invalidate any tree-level results, we will follow here the diagrammatic approach outlined in the above that provides us with the basic field theoretical picture which needs verification in an all-order QCD treatment. 
In section 2 we introduce some of the basics of TMDs needed in the rest of the paper. As said, a particularly interesting feature for the TMD soft functions entering the description of hard processes already at tree-level is the non-trivial nature of the Wilson lines connecting the non-local field operators in the matrix element. This structure of Wilson lines, arising from both collinearly and some of the transversely polarized gluon fields, now becomes dependent on the color flow in the hard process. In particular, in those situations that multiple color flow possibilities exist, this gives rise to an entanglement that can spoil factorization already at tree-level. How one gets the basic tree-level entangled result in a diagrammatic approach is outlined in section 3 .

The aim of this paper is to show that this entanglement simplifies for the 1-parton unintegrated (1PU) case, by which we refer to a situation in which only the transverse momentum in one of the hadrons is manifest. What remains is at tree-level a factorized expression with a correlator that still does have process dependence, which is contained in a non-trivial process-dependent gauge link. This is sometimes referred to as generalized factorization. The proof is given in section 4. We will also show how one can proceed if transverse parton momenta in several hadrons are involved. In such cases one can consider weighted asymmetries expressed in terms of transverse moments of the TMD functions. These weighted asymmetries actually again involve only collinear functions, but these functions are given by matrix elements of higher twist operators, among them gluonic pole matrix elements or Efremov-Teryaev-Qiu-Sterman (ETQS) functions. In section 5, we will use transverse moments to analyse single and double weighted asymmetries and illustrate this for Drell-Yan (DY) scattering and for a process with quark-quark scattering as underlying hard partonic process. The complications and non-universality always involves the gluonic pole matrix elements, which have been extensively studied [19-26]. Since such matrix elements vanish for fragmentation [27-29], we will not have to worry about the transverse momentum in the final state. We will comment on this further in our conclusions.

\section{Collinear and Transverse Momentum Dependent (TMD) correlators}

We have split up this paper in a number of sections, in which we discuss in a diagrammatic expansion the inclusion of all gluon fields that contribute at leading order and tree-level. The starting point is a hard subprocess, for which we will consider as the most generic example a two to two process with a truncated amplitude $\mathscr{M}\left(p_{1}, p_{2} ; k_{1}, k_{2}\right)$, from which the wave functions of the partons (Dirac spinors $u\left(p_{1}\right)$ for quarks, or polarizations $\epsilon\left(p_{1}\right)$ for gluons), are omitted. Rather than through wave functions, the external partons are accounted for through quark or gluon correlation or spectral functions, which are built from matrix elements of the form $\langle X|\psi(\xi)| P\rangle$ involving hadron states $|P\rangle$ rather than a free parton wave function $\langle 0|\psi(\xi)| p\rangle$. This immediately brings in the need to also consider multi-parton matrix elements with the same states, such as $\left\langle X\left|A^{\mu}(\eta) \psi(\xi)\right| P\right\rangle$.

These matrix elements appear as squared contributions in the correlators (including Dirac space indices $i$ and $j$ ),

$$
\Phi_{i j}(p ; P)=\sum_{X} \int \frac{d^{3} P_{X}}{(2 \pi)^{3} 2 E_{X}}\left\langle P\left|\bar{\psi}_{j}(0)\right| X\right\rangle\left\langle X\left|\psi_{i}(0)\right| P\right\rangle \delta^{4}\left(p+P_{X}-P\right)
$$




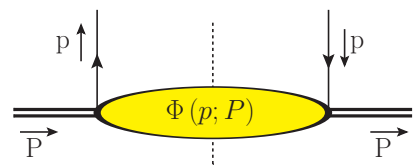

(a)

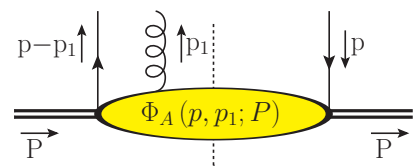

(b)

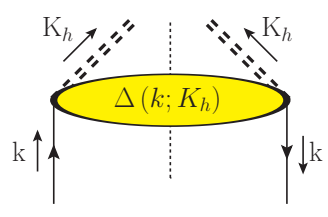

(c)

Figure 1. The pictorial momentum space representation of quark-quark correlators for distribution functions (a), a quark-quark-gluon correlator (b) and a quark-quark correlator for fragmentation functions (c).

$$
=\frac{1}{(2 \pi)^{4}} \int d^{4} \xi e^{i p \cdot \xi}\left\langle P\left|\bar{\psi}_{j}(0) \psi_{i}(\xi)\right| P\right\rangle,
$$

pictorially represented in figure 1(a). Usually, a summation over color indices is understood. This means that we will have $\Phi(p)=\operatorname{Tr}_{c}[\Phi(p)]$, where $\Phi_{i j}(p)$ is considered also a matrix in color space, made explicit $\Phi_{i j ; r s} \propto \psi_{i r}(\xi) \bar{\psi}_{j s}(0)$. Including gluon fields one has quarkquark-gluon correlators like

$$
\Phi_{A i j}^{\mu}\left(p, p_{1} ; P\right)=\frac{1}{(2 \pi)^{8}} \int d^{4} \xi d^{4} \eta e^{i\left(p-p_{1}\right) \cdot \xi} e^{i p_{1} \cdot \eta}\left\langle P\left|\bar{\psi}_{j}(0) A^{\mu}(\eta) \psi_{i}(\xi)\right| P\right\rangle,
$$

illustrated in figure 1(b), and similarly matrix elements with more partons. The color structure of the field combination $\psi_{r}(\xi) \bar{\psi}_{s}(0)$ in the quark-quark-gluon correlator now actually has a color octet structure, denoted (when appropriate) as $\Phi_{8}=\operatorname{Tr}_{c}\left[\Phi T^{a}\right] T^{a}$. Using for $A^{\mu}=A^{\mu a} T^{a}$ a matrix-valued field we have $\Phi_{A}^{\mu}=\operatorname{Tr}_{c}\left[\Phi_{8} A^{\mu}\right]$. In some cases, it will be convenient to explicitly use the momentum space fields defined as $\psi(p) \equiv \int d^{4} \xi e^{i p \cdot \xi} \psi(\xi)$ and $A_{\mu}(p) \equiv \int d^{4} \xi e^{i p \cdot \xi} A_{\mu}(\xi)$, which in the case of free fields would have parton and anti-parton contributions multiplying on-shell factors $(2 \pi) \delta\left(p^{2}-m^{2}\right) \theta\left( \pm p^{0}\right)$. For the correlators, we get

$$
\begin{aligned}
(2 \pi)^{4} \delta^{4}\left(p-p^{\prime}\right) \Phi_{i j}(p ; P) & =\frac{1}{(2 \pi)^{4}}\left\langle P\left|\bar{\psi}_{j}\left(p^{\prime}\right) \psi_{i}(p)\right| P\right\rangle \\
(2 \pi)^{4} \delta^{4}\left(p-p^{\prime}\right) \Phi_{A i j}^{\mu}\left(p, p_{1} ; P\right) & =\frac{1}{(2 \pi)^{8}}\left\langle P\left|\bar{\psi}_{j}\left(p^{\prime}\right) A^{\mu}\left(p_{1}\right) \psi_{i}\left(p-p_{1}\right)\right| P\right\rangle .
\end{aligned}
$$

The corresponding correlators describing fragmentation into hadrons is for quarks given by

$$
\begin{aligned}
\Delta_{i j}\left(k ; K_{h}\right) & =\sum_{X} \frac{1}{(2 \pi)^{4}} \int d^{4} \xi e^{-i k \cdot \xi}\left\langle 0\left|\psi_{i}(0)\right| K_{h}, X\right\rangle\left\langle K_{h}, X\left|\bar{\psi}_{j}(\xi)\right| 0\right\rangle \\
& =\frac{1}{(2 \pi)^{4}} \int d^{4} \xi e^{-i k \cdot \xi}\left\langle 0\left|\psi_{i}(0) a_{h}^{\dagger} a_{h} \bar{\psi}_{j}(\xi)\right| 0\right\rangle,
\end{aligned}
$$

pictorially represented by the blob in figure $1(\mathrm{c})$. An averaging over color indices is implicit, thus $\Delta(k)=\frac{1}{N_{c}} \operatorname{Tr}_{c}[\Delta(k)]$ with again $\Delta(k)$ a diagonal matrix in color space. The second expression in the above involves hadronic creation and annihilation operators $a_{h}^{\dagger}|0\rangle=\left|K_{h}\right\rangle$. In a momentum space representation for the operators, we have

$$
(2 \pi)^{4} \delta^{4}\left(k-k^{\prime}\right) \Delta_{i j}\left(k ; K_{h}\right)=\frac{1}{(2 \pi)^{4}} \sum_{X}\left\langle 0\left|\psi_{i}(k)\right| K_{h}, X\right\rangle\left\langle K_{h}, X\left|\bar{\psi}_{j}\left(k^{\prime}\right)\right| 0\right\rangle .
$$




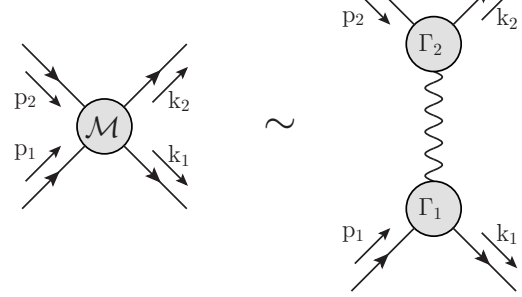

(a)

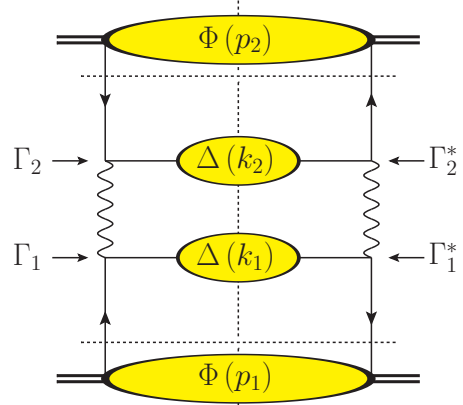

(b)

Figure 2. For the purpose of illustrating the structure of Wilson lines, we use the hard amplitude with one particular color flow for the quark lines as shown in (a). The squared amplitude needed for the cross section of the scattering process initiated by two hadrons with momenta $P_{1}$ and $P_{2}$ is shown in (b).

In fragmentation correlators, one no longer deals with plane wave hadronic states, but with out-states $\left|K_{h}, X\right\rangle$.

We want to give an expression for the cross section of the (semi)-inclusive process $H_{1}\left(P_{1}\right)+H_{2}\left(P_{2}\right) \rightarrow h_{1}\left(K_{1}\right)+h_{2}\left(K_{2}\right)+\ldots$ in a kinematic regime where $P_{1} \cdot p_{1} \sim P_{2} \cdot p_{2} \sim$ $K_{1} \cdot k_{1} \sim K_{2} \cdot k_{2}$ are small (we will refer to this scale as the squared hadronic mass scale, $M^{2} \sim 1 \mathrm{GeV}^{2}$ ) as compared to the usual hard invariants in the full or the partonic process such as $s \approx 2 P_{1} \cdot P_{2}, t_{1} \approx-2 K_{1} \cdot P_{1}, \hat{s} \approx 2 p_{1} \cdot p_{2}, \hat{t} \approx-2 k_{1} \cdot p_{1}$ (we will refer to this scale as the squared hard scale, $Q^{2} \gg M^{2}$ ). We note in passing that in cases where heavy quarks are involved, those quark masses of course have to be included. In a hard process as described here, we aim for a description in which the squared partonic amplitude $|\mathscr{M}|^{2}$ is convoluted with the correlators $\Phi(p, P), \Delta\left(k, K_{h}\right)$, etc. In order to illustrate the use of the correlators, assume an amplitude $\mathscr{M} \propto \Gamma_{1} \Gamma_{2}$ as illustrated in figure 2(a). The simplest tree-level diagrammatic contributions to the cross section that can be written down is shown in figure $2(\mathrm{~b})$ and is of the form

$$
d \sigma \sim \operatorname{Tr}_{c}\left[\Phi\left(p_{1}\right) \Gamma_{1}^{*} \Delta\left(k_{1}\right) \Gamma_{1}\right] \operatorname{Tr}_{c}\left[\Phi\left(p_{2}\right) \Gamma_{2}^{*} \Delta\left(k_{2}\right) \Gamma_{2}\right],
$$

where $\operatorname{Tr}_{c}[\ldots]$ parts are traced over color. This expression still needs to be integrated over the parton momenta, which will be discussed below. In the case that the vertices $\Gamma$ don't have any color structure, one can, because of the simple color singlet structure of $\Phi$ and $\Delta$ in the quark-quark correlators, perform the color trace separately for $\Phi$ and $\Delta$, $\operatorname{Tr}_{c}\left[\Phi(p) \Gamma^{*} \Delta(k) \Gamma\right]=\operatorname{Tr}_{c}[\Phi(p)] \frac{1}{N_{c}} \operatorname{Tr}_{\mathrm{c}}[\Delta(k)] \Gamma \Gamma^{*}$ (one summed and one averaged) and the cross section can be written in terms of the color-traced entities

$$
d \sigma \sim \Phi\left(p_{1}\right) \Phi\left(p_{2}\right) \underbrace{\Gamma_{1} \Gamma_{1}^{*} \Gamma_{2} \Gamma_{2}^{*}}_{\hat{\Sigma}} \Delta\left(k_{1}\right) \Delta\left(k_{2}\right),
$$

where the remaining contractions are Dirac space and Lorentz indices, which have been suppressed in both eqs. (2.7) and (2.8). These expressions of course should be extended 
with all possible correlators containing quark and gluon fields, in which cases color traces become more complicated because the quark-quark part of the correlator can have a color octet structure $\left(\Phi_{8}\right)$. The restriction to hard kinematics limits the number of diagrammatic contributions, although even at leading order, there still are many gluon contributions as will be discussed in section 3 . These will complicate the color tracing, an effect which is particularly important when dependence on transverse momenta is considered, which precisely is our goal in this paper. We will only work out leading contributions in an $M / Q$ expansion, although the separation of various orders requires care, as we will argue in section 3. The $M^{2} / Q^{2}$ effects certainly cannot be calculated in our diagrammatic approach. At that level, there are many contributions that spoil already at tree-level the possibility to write down in a consistent way a cross section in the form of eq. (2.7) and certainly to perform the color traces as in eq. (2.8).

For parton momenta relevant in a hadron correlator (hadron momentum $P$ ) we make the Sudakov decomposition,

$$
p=x P+p_{T}+\underbrace{\left(p \cdot P-x M^{2}\right)}_{\sigma} n,
$$

where the role of the (approximately) light-like vector $n$, satisfying $P \cdot n=1$ can come from any of the hard (external) momenta, e.g. $n=K_{h} / K_{h} \cdot P$ or $n=k / k \cdot P$ (provided $\left.k \cdot P \sim K_{h} \cdot P \sim Q^{2}\right)$. The momentum fraction $x=p \cdot n=p^{n}$ is $\mathscr{O}(1)$. For any contractions with vectors outside the correlator $\Phi(p, P)$ one has $P \sim Q, p_{T} \sim M$ and $n \sim 1 / Q$. Note that if $n$ is an exact light-like vector, one can construct two exact conjugate null-vectors,

$$
n_{+}=P-\frac{1}{2} M^{2} n \quad \text { and } \quad n_{-}=n,
$$

satifying $n_{+} \cdot n_{-}=1$ and $n_{+}^{2}=n_{-}^{2}=0$, that can be used to define light-cone components $a^{ \pm}=a \cdot n_{\mp}$ (thus $x=p \cdot n_{-}=p^{+}$). The symmetric and antisymmetric 'transverse' projectors are defined as

$$
\begin{aligned}
& g_{T}^{\mu \nu}=g^{\mu \nu}-n_{+}^{\{\mu} n_{-}^{\nu\}}=g^{\mu \nu}-P^{\{\mu} n^{\nu\}}+M^{2} n^{\mu} n^{\nu} \approx g^{\mu \nu}-P^{\{\mu} n^{\nu\}}, \\
& \epsilon_{T}^{\mu \nu}=\epsilon^{n_{+} n_{-} \mu \nu}=\epsilon^{-+\mu \nu}=\epsilon^{P n \mu \nu} .
\end{aligned}
$$

Since transverse momentum dependence is a central issue in this paper, we have to worry about different $n$ vectors. With $\Delta n=n^{\prime}-n \sim 1 / Q$ one has at $\mathscr{O}\left(Q^{0}\right)$ that $\Delta x \approx \Delta p_{T}^{2} \approx 0$ although the transverse momentum itself does change at order $\mathscr{O}(1), \Delta p_{T}=-\Delta x P$.

The integration over parton momenta,

$$
\int d^{4} p=\int d x d^{2} p_{T} d \sigma=\int d(p \cdot n) d^{2} p_{T} d(p \cdot P),
$$

is insensitive to the particular $n$ vector. In view of the relative importance of the components in this integration, one can, upon neglecting any $M^{2} / Q^{2}$ contributions in the cross section, integrate within a soft correlator over $p \cdot P$ (i.e. $p^{-}$) to obtain the TMD correlator

$$
\Phi\left(x, p_{T} ; n\right)=\int d p \cdot P \Phi(p ; P)=\left.\int \frac{d \xi \cdot P d^{2} \xi_{T}}{(2 \pi)^{3}} e^{i p \cdot \xi}\langle P|\bar{\psi}(0) \psi(\xi)| P\rangle\right|_{L F}
$$


which we will still consider as the unintegrated correlator. On the left-hand side the dependence on hadron momentum $P$ has been suppressed. In the TMD correlator the non-locality is restricted to the light-front (LF: $\left.\xi \cdot n=\xi^{+}=0\right)$ and the correlator depends on $x=p \cdot n$ and $p_{T}$. The light-cone correlators are the collinear correlators containing the parton distribution functions depending only on the light-cone momentum fraction $x$, obtained upon integration over both $p \cdot P$ and $p_{T}$,

$$
\Phi(x ; n)=\int d p \cdot P d^{2} p_{T} \Phi(p ; P)=\left.\int \frac{d \xi \cdot P}{(2 \pi)} e^{i p \cdot \xi}\langle P|\bar{\psi}(0) \psi(\xi)| P\rangle\right|_{L C}
$$

where the subscript LC refers to light-cone, implying $\xi \cdot n=\xi_{T}=0$. This integration is generally allowed in hard processes up to $M^{2} / Q^{2}$ contributions and also up to contributions coming from the tails, e.g. logarithmically divergent contributions proportional to $\alpha_{s}\left(p_{T}^{2}\right) / p_{T}^{2}$ tails [30] and relevant when looking at evolution [31]. Such contributions, however, require next-to-leading order (NLO) QCD, which goes beyond the tree-level resummations that we discuss in this paper. In diagrammatic language they for instance involve ladder graphs describing emission of gluons into the final state, relevant for the evolution of the correlators. The collinear correlators are relevant in hard processes in which only hard scales (large invariants $\sim Q^{2}$ or ratios thereof, angles, rapidities) are measured. If one considers hadronic scale observables (correlations or transverse momenta in jets, slightly off-collinear configurations) one will need the TMD correlators for a full treatment.

The correlators encompass the information on the soft parts. They depend on the hadron and quark momenta $P$ and $p$ (and in general also spin vectors). Depending on the Lorentz and Dirac structure of the matrix elements involved one can look for the pieces in the correlator that show up as the most dominant matrix elements among the contributions in the hard process. Including also gluon fields, the Fourier transform of matrix elements with a maximal number of contractions with $n$,

$$
\langle\bar{\psi}(0) \not h \psi(\xi)\rangle \quad \text { and } \quad\left\langle G^{n \alpha}(0) G^{n \beta}(\xi)\right\rangle
$$

(the latter with transverse indices $\alpha$ and $\beta$ ) are the dominant combinations that appear in the correlators. They are the dominant ones because the contractions with $n$ lower the canonical dimension of the operator combination, minimizing the power of $M$ that after contractions of open indices inevitably is the scale of the hadronic matrix elements. The two matrix elements above have canonical dimension two. The corresponding local matrix elements, $\bar{\psi}(0) \not h \psi(0)$ and $G^{n \alpha}(0) G^{n \beta}(0)$ for quarks and gluons, respectively, are color gauge-invariant (twist 2) operators, the non-local combinations in eq. (2.16) are not gauge invariant. Expanded into local operators, the expansion would involve operator combinations with derivatives such as $\bar{\psi}(0) \not \partial^{n} \ldots \partial^{n} \psi(0)$. Color gauge invariance in the correlators requires in the local matrix elements covariant derivatives or in the non-local matrix elements the presence of a gauge link connecting the two fields. For the light-cone correlators the gauge link corresponds to the inclusion of arbitrary number of 'leading' gluon fields $A^{n}(\eta)$ in the field combinations in eq. (2.16) which are resummed into a gauge 
$\operatorname{link} \bar{\psi}(0) W_{[0, \xi]}^{[n]} \psi(\xi)=\operatorname{Tr}_{c}\left[W_{[0, \xi]}^{[n]} \psi(\xi) \bar{\psi}(0)\right]$, given by

$$
W_{[0, \xi]}^{[n]}=\mathscr{P} \exp \left(-i \int_{0}^{\xi} d \eta \cdot P n \cdot A(\eta)\right) .
$$

Including this gauge link, the non-local operator combinations

$$
\left.\left\langle\bar{\psi}(0) \not h W_{[0, \xi]}^{[n]} \psi(\xi)\right\rangle\right|_{L C} \quad \text { and }\left.\quad\left\langle G^{n \alpha}(0) W_{[0, \xi]}^{[n]} G^{n \beta}(\xi) W_{[\xi, 0]}^{[n]}\right\rangle\right|_{L C},
$$

can be expanded into twist two operators $\bar{\psi}(0) \not h D^{n} \ldots D^{n} \psi(0)$ and $G^{n \alpha}(0) D^{n} \ldots D^{n} G^{n \beta}(0)$ for quarks and gluons, respectively (number of $D^{n}$ 's is the spin of these operators). Also TMD correlators require a gauge link, but the separation of the two fields is no longer a simple light-like one and they involve derivatives with transverse indices. It is important to realize that in principle any gauge link with an arbitrary path gives a gauge-invariant combination. What is the appropriate link contributing at leading order (in $M / Q$ ) in a given hard scattering process, however, is calculable (see next section).

The correlators in this section have been rewritten into matrix elements of non-local products of fields. They involve both quark and gluon fields as well as hadronic states or hadronic creation and annihilation operators. For them there is no systematic perturbative expansion in terms of the strong coupling constant. The kinematic separation of soft and hard, however, allows the integration over $p^{-}=p \cdot P$, leaving a product of operators at the light-front, that is at equal light-cone time $\xi^{+}=\xi \cdot n=0$. For such a product the time-ordering is automatic, which means that the $p^{-}$-integrated parton correlators, thus, can be considered as a cut anti-parton-hadron scattering amplitude, i.e. a Green function, untruncated in the parton legs [32]. This is the case for both collinear and TMD correlators [33]. This identification has been very important in deep inelastic processes [34], allowing the use of analyticity and unitarity properties of field theories, at least under the assumption that these properties apply to QCD. We will use it later in this paper for fragmentation correlators.

\section{Color gauge invariance}

It is worthwhile to repeat the steps that lead to color gauge invariance by including collinear gluon fields, which will also be the first step to obtain the Wilson lines in TMD correlators. We include in the diagrammatic approach matrix elements with gluon fields for which we make a Sudakov expansion,

$$
A^{\mu}(\eta)=A^{n}(\eta) P^{\mu}+A_{T}^{\mu}(\eta)+\left(A^{P}(\eta)-A^{n}(\eta) M^{2}\right) n^{\mu} .
$$

A similar expansion can be written down for $A^{\mu}(p)$. In order to use Ward identities it will be convenient to look at the (collinear) gluon field component along parton momentum $p^{\mu}$, hence we write

$$
\begin{aligned}
A^{\mu}(p) & =\int d^{4} \eta e^{i p \cdot \eta} A^{\mu}(\eta) \\
& =\int d^{4} \eta e^{i p \cdot \eta}\left[\frac{A^{n}(\eta)}{p \cdot n} p^{\mu}+\frac{(p \cdot n) A_{T}^{\mu}(\eta)-p_{T}^{\mu} A^{n}(\eta)}{p \cdot n}+\frac{(p \cdot n) A^{P}(\eta)-(p \cdot P) A^{n}(\eta)}{p \cdot n} n^{\mu}\right] .
\end{aligned}
$$




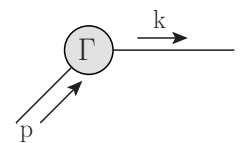

$A_{0}$

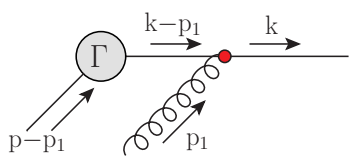

$A_{1}$

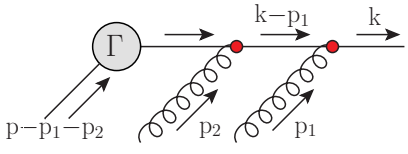

$A_{2}$

Figure 3. Inclusion of collinear gluons from $\Phi_{A \ldots A}\left(p-p_{1} \ldots-p_{N}, p_{1}, \ldots, p_{N}\right)$ coupling to an outgoing (colored) quark line with momentum $k$.

In the correlator the momentum $p^{\mu} \longrightarrow i \partial^{\mu}(\eta)$, so

$$
\begin{aligned}
& A^{\mu}(p)=\frac{1}{p \cdot n} \int d^{4} \eta e^{i p \cdot \eta}\left[A^{n}(\eta) p^{\mu}+i \partial^{n}(\eta) A_{T}^{\mu}(\eta)-i \partial_{T}^{\mu}(\eta) A^{n}(\eta)\right. \\
& \left.+\left(i \partial^{n}(\eta) A^{P}(\eta)-i \partial^{P}(\eta) A^{n}(\eta)\right) n^{\mu}\right] \\
& =\frac{1}{p \cdot n}\left[A^{n}(p) p^{\mu}+i G_{T}^{n \mu}(p)+i G^{n P}(p) n^{\mu}\right] \text {. }
\end{aligned}
$$

Although the latter appears to be only true for the Abelian case, we will find the same result in the non-abelian case, but to complete that proof, we first need to incorporate the collinear gluons into the matrix elements. Using the expansion in eq. (3.4) rather than the one in eq. (3.1) streamlines the inclusion of collinear gluons circumventing the explicit treatment of transverse momentum dependent parts (as done in ref. [9]). The results are of course identical.

\subsection{Collinear gluons}

Since matrix elements involving operator combinations $\bar{\psi}(0) A^{n}\left(\eta_{1}\right) \ldots A^{n}\left(\eta_{N}\right) \psi(\xi)$ and $G^{n \alpha}(0) A^{n}\left(\eta_{1}\right) \ldots A^{n}\left(\eta_{N}\right) G^{n \beta}(\xi)$ for quarks and gluons are as leading as the matrix elements without collinear gluons, the contributions of correlators with gluon fields of which the polarization is along its momentum $p^{\mu}$, i.e. the first term in eq. (3.4) need to be included in the leading expression for the cross section. Using Ward identities, only the contributions of gluons coupling to external parton lines survive as discussed in detail in ref. [11]. The resummation is best illustrated by looking at the example of gluons attached to a $\Phi(p)$ correlator attached to an outgoing quark line with momentum $k$ (see figure 3 ). The results of these parts within the amplitude are

$$
A_{0}=\bar{\psi}(k) \Gamma \psi(p)
$$

where the $\psi(p)$ and $\bar{\psi}(k)$ are fields belonging to the correlators of initial (momentum $p$ ) and final state quark (momentum $k$ ) respectively. The one-gluon contribution to the link is

$$
A_{1}=\int \frac{d^{4} p_{1}}{(2 \pi)^{4}} \bar{\psi}(k) \frac{-i \not p_{1} A^{k}\left(p_{1}\right)}{p_{1} \cdot k} \frac{i\left(k-\not p_{1}\right)}{\left(-2 k \cdot p_{1}+i \epsilon\right)} \Gamma \psi\left(p-p_{1}\right) .
$$


The numerator becomes $\not p_{1}\left(\not k-\not p_{1}\right)=\not \not_{1} \not k=\left\{\not k, \not \not_{1}\right\}=2 k \cdot p_{1}$. The added term is zero since $\bar{\psi}(k) \not k \approx 0$. Thus one has (note that the sign of $k \cdot p_{1}$ is positive),

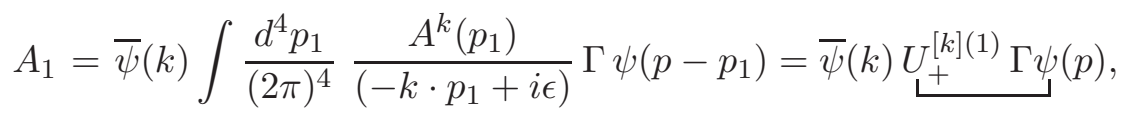

which is the one-gluon contribution to a path-ordered exponential (see appendix A). The two-gluon term becomes

$$
\begin{aligned}
A_{2}= & \int \frac{d^{4} p_{1}}{(2 \pi)^{4}} \frac{d^{4} p_{2}}{(2 \pi)^{4}} \bar{\psi}(k) \frac{A^{k}\left(p_{1}\right)}{p_{1} \cdot k} \frac{A^{k}\left(p_{2}\right)}{p_{2} \cdot k} \not p_{1} \frac{1}{\not k-\not p_{1}} \not p_{2} \frac{1}{\not k-\not p_{1}-\not p_{2}} \Gamma \psi\left(p-p_{1}-p_{2}\right) \\
= & \int \frac{d^{4} p_{1}}{(2 \pi)^{4}} \frac{d^{4} p_{2}}{(2 \pi)^{4}} \bar{\psi}(k) \frac{A^{k}\left(p_{1}\right)}{p_{1} \cdot k} \frac{A^{k}\left(p_{2}\right)}{p_{2} \cdot k} \frac{\not p_{1}\left(\not k-\not p_{1}\right)}{\left(-2 k \cdot p_{1}+i \epsilon\right)} \\
& \times \frac{\not p_{2}\left(\not k-\not p_{1}-\not p_{2}\right)}{\left(-2 k \cdot p_{1}-2 k \cdot p_{2}+i \epsilon\right)} \Gamma \psi\left(p-p_{1}-p_{2}\right) \\
= & \bar{\psi}(k) \int \frac{d^{4} p_{1}}{(2 \pi)^{4}} \frac{d^{4} p_{2}}{(2 \pi)^{4}} \frac{A^{k}\left(p_{1}\right)}{\left(-k \cdot p_{1}+i \epsilon\right)} \frac{A^{k}\left(p_{2}\right)}{\left(-k \cdot p_{1}-k \cdot p_{2}+i \epsilon\right)} \Gamma \psi\left(p-p_{1}-p_{2}\right) \\
= & \bar{\psi}(k) U_{+}^{[k](2)} \Gamma \psi(p) .
\end{aligned}
$$

This is the two-gluon contribution to the path-ordered exponential. This term illustrates the recursive procedure that gives the all-gluon results to the full path-ordered exponential or Wilson line (see appendix A),

$$
\sum_{N=0}^{\infty} A_{N}=\bar{\psi}(k) \underbrace{U_{+}^{[k]} \Gamma \psi}_{+}(p),
$$

where

$$
\underset{+}{U_{+}^{[n]} \psi} \psi(p)=\int d^{4} \xi \exp (i p \cdot \xi) \mathscr{P} \exp \left(-i g \int_{\infty}^{\xi \cdot P} d(\eta \cdot P) A^{n}(\eta)\right) \psi(\xi)
$$

is the Fourier transform of the field including the Wilson line of which the indices of $U_{+}^{[n]}$ indicate its direction, namely a Wilson line running from light-cone $+\infty$ to $\xi$ along the light-like direction $n$. We will instead of the notation with the contraction, mostly use the notation

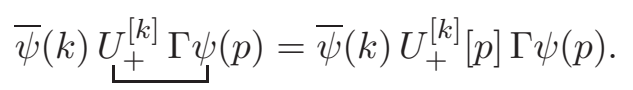

The second expression gives the Wilson line a label $[p]$, indicating that all fields in it belong to the correlator $\Phi(p)$, to which also $\psi(p)$ belongs. The problem with this path-ordered exponential is that it is a unitary matrix in color space that is stuck in the respective traces, although it is in fact only the (symmetric) color charge operators $T^{a_{1}} \ldots T^{a_{s}}$ of the terms $U_{+}^{[n](s)}$ in the expansion of the $U_{+}^{[n]}$ that are stuck there.

For the gluon insertions coming from a particular correlator and coupling to an incoming fermion line one finds a Wilson line connecting to light-cone $-\infty$, which is a consequence 


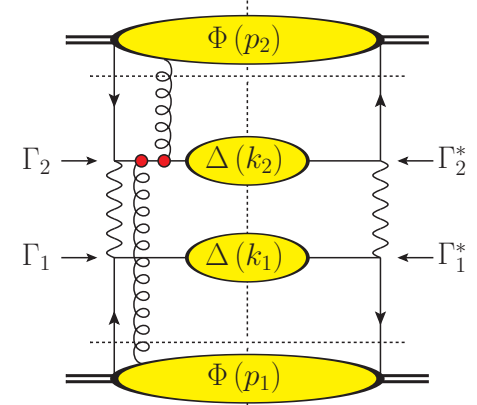

(a)

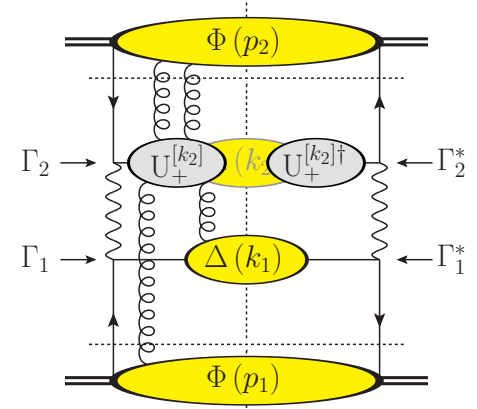

(b)

Figure 4. (a) An example of a diagram with two gluons attaching to the same outgoing line with momentum $k_{2}$. (b) In more detail these couplings are discussed in the appendices and they result into one gauge connection $U_{+}^{\left[k_{2}\right]}\left[p_{1}, p_{2}, k_{1}\right]$, which combines all collinear gluons coming from $\Phi\left(p_{1}\right)$, $\Phi\left(p_{2}\right)$ and $\Delta\left(k_{1}\right)$. Together with transverse pieces, and combining it with gauge connections at other legs one will get a full color gauge invariant result, discussed in section 3 .

of the sign $k \cdot p_{i}$ being negative in that situation. Including all multi-gluon interactions originating from $\Phi\left(p_{1}\right)$ in figure 2(b), we get the diagrammatic result

$$
\begin{aligned}
d \sigma \sim & \operatorname{Tr}_{c}\left[\Phi\left(p_{1}\right) \Gamma_{1}^{*} U_{+}^{\left[k_{1}\right] \dagger}\left[p_{1}\right] \Delta\left(k_{1}\right) U_{+}^{\left[k_{1}\right]}\left[p_{1}\right] \Gamma_{1}\right] \\
& \times \operatorname{Tr}_{c}\left[U_{-}^{\left[p_{2}\right] \dagger}\left[p_{1}\right] \Phi\left(p_{2}\right) U_{-}^{\left[p_{2}\right]}\left[p_{1}\right] \Gamma_{2}^{*} U_{+}^{\left[k_{2}\right] \dagger}\left[p_{1}\right] \Delta\left(k_{2}\right) U_{+}^{\left[k_{2}\right]}\left[p_{1}\right] \Gamma_{2}\right],
\end{aligned}
$$

with the (color charge of the) Wilson line stuck in the color traces at the 'positions' corresponding to the external parton lines. Note that in eq. (3.10) the Wilson lines in sub-expressions like $U_{+}^{\left[k_{1}\right] \dagger}\left[p_{1}\right] \Delta\left(k_{1}\right) U_{+}^{\left[k_{1}\right]}\left[p_{1}\right]$ are part of the Fourier transform in correlator $\Phi\left(p_{1}\right)$. In coordinate space, taking $\xi_{1}$ to be the coordinate conjugate to $p_{1}$, the link and conjugate link actually run between different points, in this case $U_{+}$in $U_{+}^{\left[k_{1}\right]}\left[p_{1}\right] \ldots \psi\left(p_{1}\right)$ corresponds actually to $U_{\left[+\infty, \xi_{1}\right]}^{\left[k_{1}\right]}$, while the conjugate link $U_{+}^{\dagger}$ in $\bar{\psi}\left(p_{1}\right) \ldots U_{+}^{\left[k_{1}\right] \dagger}\left[p_{1}\right]$ corresponds to $U_{\left[0_{1},+\infty\right]}^{\left[k_{1}\right]}$. Wilson lines between $0_{1 T}$ and $\xi_{1 T}$ are still lacking, so eq. (3.10) is certainly not color gauge-invariant.

The next step in our treatment is the inclusion of gluon interactions coming from different correlators, say $\Phi\left(p_{1}\right)$ and $\Phi\left(p_{2}\right)$, coupling to the same quark line with momentum $k_{2}$, such as shown e.g. in figure $4(\mathrm{a})$. They give rise to intertwined Wilson lines. Two examples of this have been given in appendix B, for the case of two gluons coming from different correlators and for the case of three gluons, two coming from the same correlator and one from a different correlator. These examples illustrate the recursive procedure. The result for all insertions to a particular leg is a color symmetric combination of the insertions from all correlators, which since all gluon polarizations are identical is just

$$
U_{+}^{\left[k_{2}\right]}\left[p_{1}, p_{2}, k_{1}\right]=\mathcal{S}\left\{U_{+}^{\left[k_{2}\right]}\left[p_{1}\right] U_{+}^{\left[k_{2}\right]}\left[p_{2}\right] U_{+}^{\left[k_{2}\right]}\left[k_{1}\right]\right\},
$$

in which the ordering of the three connections on the right-hand side is irrelevant (fully symmetrized). This is illustrated in figure 4. Including all multi-gluon interactions from 
$\Phi\left(p_{1}\right), \Delta\left(k_{1}\right), \Phi\left(p_{2}\right)$ and $\Delta\left(k_{2}\right)$ onto all legs, eq. (3.10) generalizes to

$$
\begin{aligned}
d \sigma \sim & \operatorname{Tr}_{c}\left[U_{-}^{\left[p_{1}\right] \dagger}\left[p_{2}, k_{1}, k_{2}\right] \Phi\left(p_{1}\right) U_{-}^{\left[p_{1}\right]}\left[p_{2}, k_{1}, k_{2}\right] \Gamma_{1}^{*} U_{+}^{\left[k_{1}\right] \dagger}\left[p_{1}, p_{2}, k_{2}\right] \Delta\left(k_{1}\right) U_{+}^{\left[k_{1}\right]}\left[p_{1}, p_{2}, k_{2}\right] \Gamma_{1}\right] \\
& \times \operatorname{Tr}_{c}\left[U_{-}^{\left[p_{2}\right] \dagger}\left[p_{1}, k_{1}, k_{2}\right] \Phi\left(p_{2}\right) U_{-}^{\left[p_{2}\right]}\left[p_{1}, k_{1}, k_{2}\right] \Gamma_{2}^{*} U_{+}^{\left[k_{2}\right] \dagger}\left[p_{1}, p_{2}, k_{1}\right] \Delta\left(k_{2}\right) U_{+}^{\left[k_{2}\right]}\left[p_{1}, p_{2}, k_{1}\right] \Gamma_{2}\right],
\end{aligned}
$$

illustrated in figure 5(a). We note that the Wilson lines in eq. (3.12) have different light-like directions, which originate from the fact that we in the decomposition of gluon fields in eq. (3.4) simply made the most convenient choice depending on the particular correlator. For two different Sudakov decompositions of the gluon field, one finds that

$$
\frac{A^{n}(p)}{p \cdot n}-\frac{A^{n^{\prime}}(p)}{\left(p \cdot n^{\prime}\right)}=\frac{i G^{n n^{\prime}}(p)}{(p \cdot n)\left(p \cdot n^{\prime}\right)} .
$$

The field $G^{n n^{\prime}}(p)$, however, appearing in a correlator $\Phi_{G}^{n n^{\prime}}$ will not contribute at leading order, but at subleading order $(1 / Q)$. This allows one to replace all the light-like direction dependence in eq. (3.12) by a generic null-vector $n$. Even after choosing one light-like direction, the result in eq. (3.12) is not yet gauge-invariant, since transverse gauge connections are still missing.

\subsection{Collinear correlators}

The missing transverse pieces don't matter when one takes a collinear approach, implying integration over transverse momenta $p_{T}$ besides the integration over $p \cdot P$. In that case there is no transverse separation of the fields. After integration over transverse momenta, one has a color gauge-invariant result,

$$
\begin{aligned}
\sigma \sim \operatorname{Tr}_{c}\left[U_{-}^{[n] \dagger}\left[p_{2}, k_{1}, k_{2}\right] \Phi\left(x_{1}\right) U_{-}^{[n]}\left[p_{2}, k_{1}, k_{2}\right] \Gamma_{1}^{*} U_{+}^{[n] \dagger}\left[p_{1}, p_{2}, k_{2}\right] \Delta\left(z_{1}\right) U_{+}^{[n]}\left[p_{1}, p_{2}, k_{2}\right] \Gamma_{1}\right] \\
\quad \times \operatorname{Tr}_{c}\left[U_{-}^{[n] \dagger}\left[p_{1}, k_{1}, k_{2}\right] \Phi\left(x_{2}\right) U_{-}^{[n]}\left[p_{1}, k_{1}, k_{2}\right] \Gamma_{2}^{*} U_{+}^{[n] \dagger}\left[p_{1}, p_{2}, k_{1}\right] \Delta\left(z_{2}\right) U_{+}^{[n]}\left[p_{1}, p_{2}, k_{1}\right] \Gamma_{2}\right] .
\end{aligned}
$$

One can combine the Wilson lines to and from light-cone $\pm \infty$, all made up of $A^{n}$ fields, into finite Wilson lines, e.g. $W^{[n]}\left[p_{1}\right]=U_{+}^{[n] \dagger}\left[p_{1}\right] U_{+}^{[n]}\left[p_{1}\right]$ since after the integration over $p_{1 T}$ they not only both run along $n$, but they coincide since one also has $0_{T}=\xi_{T}$. Furthermore, it is irrelevant if one composes $W^{[n]}$ from Wilson lines running via plus or via minus infinity, and also the direction $n$ is in fact irrelevant. It is just the direction of the straight line connecting 0 and $\xi$. We recall that the argument $p_{1}$ or $x_{1}$, given to the Wilson lines, is simply needed to indicate that the fields in that Wilson line belong to the correlator $\Phi\left(x_{1}\right)$, which is the Fourier transform of the matrix element $\langle\bar{\psi}(0) \psi(\xi)\rangle$. Thus in coordinate space one just has the Wilson line in eq. (2.17), which connects the points 0 and $\xi$ in $\Phi\left(x_{1}\right)$ composed of $W_{[0, \xi]}^{[n]}=U_{[0, \infty]}^{[n]} U_{[\infty, \xi]}^{[n]}$. As far as relevant for $\Phi\left(x_{1}\right)$, the Wilson lines in the first trace form a gauge link, those in the second trace form a closed loop, which in the collinear situation (when $0_{T}=\xi_{T}$ ) becomes a unit operator in color space. One is left with

$$
\begin{aligned}
\sigma \sim & \operatorname{Tr}_{c}\left[U_{-}^{[n] \dagger}\left[k_{1}\right] \Phi\left(x_{1}\right) U_{-}^{[n]}\left[k_{1}\right] \Gamma_{1}^{*} U_{-}^{[n] \dagger}\left[p_{1}\right] \Delta\left(z_{1}\right) U_{-}^{[n]}\left[p_{1}\right]\right] \\
& \times \operatorname{Tr}_{c}\left[U_{-}^{[n] \dagger}\left[k_{2}\right] \Phi\left(x_{2}\right) U_{-}^{[n]}\left[k_{2}\right] \Gamma_{2}^{*} U_{-}^{[n] \dagger}\left[p_{2}\right] \Delta\left(z_{2}\right) U_{-}^{[n]}\left[p_{2}\right] \Gamma_{2}\right] .
\end{aligned}
$$


The way of turning the gauge connections into gauge links at the collinear stage is actually just applying gauge transformations $U_{[a, \xi]}^{[n]}$ (with a fixed point $a$ ) to all fields. This can actually directly be applied to eq. (3.14). One obtains

$$
\sigma \sim \Phi^{[W]}\left(x_{1}\right) \Phi^{[W]}\left(x_{2}\right) \underbrace{\Gamma_{1} \Gamma_{1}^{*} \Gamma_{2} \Gamma_{2}^{*}}_{\hat{\Sigma}} \Delta^{[W]}\left(z_{1}\right) \Delta^{[W]}\left(z_{2}\right),
$$

where

$$
\begin{aligned}
\Phi^{[W]}(x) & =\operatorname{Tr}_{c}\left[U_{ \pm}^{[n]}[p] \Phi(x) U_{ \pm}^{[n] \dagger}[p]\right]=\left.\int \frac{d \xi \cdot P}{2 \pi} e^{i p \cdot \xi}\left\langle P\left|\bar{\psi}(0) U_{[0, \pm \infty]}^{[n]} U_{[ \pm \infty, \xi]}^{[n]} \psi(\xi)\right| P\right\rangle\right|_{L C} \\
& =\operatorname{Tr}_{c}\left[W^{[n]}[p] \Phi(x)\right]=\left.\int \frac{d \xi \cdot P}{2 \pi} e^{i p \cdot \xi}\left\langle P\left|\bar{\psi}(0) W_{[0, \xi]}^{[n]} \psi(\xi)\right| P\right\rangle\right|_{L C}
\end{aligned}
$$

and

$$
\begin{aligned}
\Delta^{[W]}(z) & =\frac{1}{N_{c}} \operatorname{Tr}_{c}\left[U_{ \pm}^{[n]}[k] \Delta(z) U_{ \pm}^{[n] \dagger}[k]\right]=\frac{1}{N_{c}} \operatorname{Tr}_{c}\left[\Delta(z) W^{[n] \dagger}[k]\right] \\
& =\left.\int \frac{d \xi \cdot K_{h}}{2 \pi} e^{-i k \cdot \xi} \frac{1}{N_{c}} \operatorname{Tr}_{c}\left\langle 0\left|U_{[ \pm \infty, 0]}^{[n]} \psi(0) a_{h}^{\dagger} a_{h} \bar{\psi}(\xi) U_{[\xi, \pm \infty]}^{[n]}\right| 0\right\rangle\right|_{L C}
\end{aligned}
$$

are the color gauge-invariant collinear correlators, including unique gauge links $W$ along the light-like separation. The gauge link being unique, it is usually omitted. These (color gauge-invariant) correlators can be expanded in terms of the standard parton distribution functions and fragmentation functions, respectively.

For the correlator $\Phi(x)$ it is also possible to circumvent manipulating with Wilson lines by taking moments in $x_{1}=p_{1} \cdot n, x_{2}=p_{2} \cdot n, 1 / z_{1}=k_{1} \cdot n$ and $1 / z_{2}=k_{2} \cdot n$. Using

$$
i \partial_{\xi}^{n} W_{[\eta, \xi]}^{[n]}=W_{[\eta, \xi]}^{[n]} i D^{n}(\xi),
$$

one easily verifies the factorized expression in eq. (3.16). In the process of taking moments one then encounters $x^{N} \Phi^{[W]}(x)$ involving matrix elements with covariant derivatives $D^{n}$,

$$
x^{N} \Phi^{[W]}(x)=\left.\int \frac{d \xi \cdot P}{2 \pi} e^{i p \cdot \xi}\left\langle P\left|\bar{\psi}(0) W_{[0, \xi]}^{[n]}\left(i D^{n}\right)^{N} \psi(\xi)\right| P\right\rangle\right|_{L C} .
$$

We have shown everything for one correlator, but one has similar expressions for the other correlators. Since the gauge link is unique for the collinear correlators, we will just write $\Phi\left(x_{1}\right), \Phi\left(x_{2}\right), \Delta\left(z_{1}\right)$ and $\Delta\left(z_{2}\right)$.

\subsection{Including transverse gauge connections}

To see how transverse gauge connections arise, we jump back to eq. (3.12) and note that not only the $\langle\ldots \bar{\psi}(0) \psi(\xi) \ldots\rangle$ correlator has acquired gauge connections along the $n$-direction, but also other matrix elements involving the other gluon components in eq. (3.3), e.g. the operator combination

$$
\left\langle\ldots i \partial^{n}(\eta) U_{[\infty, \eta]}^{[n]} A_{T}^{\alpha}(\eta) U_{[\eta, \infty]}^{[n]}-i \partial_{T}^{\alpha}(\eta) U_{[\infty, \eta]}^{[n]} A^{n}(\eta) U_{[\eta, \infty]}^{[n]} \ldots\right\rangle,
$$




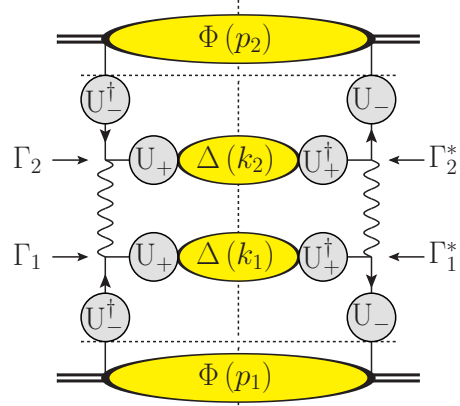

(a)

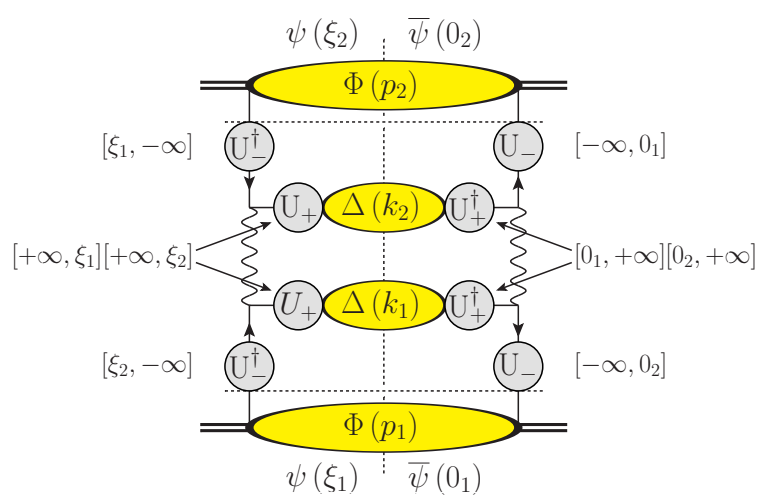

(b)

Figure 5. (a) The gauge connections from all collinear gluons from the various soft correlators produce (entangled) gauge connections with color charges located on the external legs of the hard part. (b) We have indicated for the correlators and gauge connections also the actual space-time points they are bridging limiting ourselves for simplicity to the coordinates conjugate to $p_{1}$ (points $0_{1}$ and $\xi_{1}$; see also the discussion following eq. (3.10)) and $p_{2}$ (points $0_{2}$ and $\xi_{2}$ ), leaving out the space-time structure for the fragmentation correlators for which we would have to include also the coordinates conjugate to $k_{1}$ and $k_{2}$.

for which we can use the non-abelian relation (directly based on eq. (3.19)),

$$
\begin{aligned}
U_{[\infty, \eta]}^{[n]} A_{T}^{\alpha}(\eta) & U_{[\eta, \infty]}^{[n]}-\left.A_{T}^{\alpha}(\eta)\right|_{\eta^{P}=\infty} \\
& =\left.\int_{\infty}^{\eta^{P}} d \zeta^{P} U_{[\infty, \zeta]}^{[n]}\left(G^{n \alpha}(\zeta)+\left[\partial_{T}^{\alpha}, A^{n}(\zeta)\right]\right) U_{[\zeta, \infty]}^{[n]}\right|_{\zeta^{n}=\eta^{n}, \zeta_{T}=\eta_{T}} .
\end{aligned}
$$

Differentiation with respect to $\partial_{\eta}^{n}=\partial / \partial \eta^{P}$ gives

$$
\begin{gathered}
\left\langle\ldots i \partial^{n}(\eta) U_{[\infty, \eta]}^{[n]} A_{T}^{\alpha}(\eta) U_{[\eta, \infty]}^{[n]}-i \partial_{T}^{\alpha}(\eta) U_{[\infty, \eta]}^{[n]} A^{n}(\eta) U_{[\eta, \infty]}^{[n]} \ldots\right\rangle \\
=\left\langle\ldots i U_{[\infty, \eta]}^{[n]} G_{T}^{n \alpha}(\eta) U_{[\eta, \infty]}^{[n]} \ldots\right\rangle
\end{gathered}
$$

showing that the transition of eq. (3.3) to eq. (3.4) also works in the non-abelian case, if the appropriate Wilson lines are included. Similarly, one has

$$
\begin{aligned}
U_{[\infty, \eta]}^{[n]} A^{P}(\eta) & U_{[\eta, \infty]}^{[n]}-\left.A^{P}(\eta)\right|_{\eta^{P}=\infty} \\
& =\left.\int_{\infty}^{\eta^{P}} d \zeta^{P} U_{[\infty, \zeta]}^{[n]}\left(G^{n P}(\zeta)+\left[\partial^{P}, A^{n}(\zeta)\right]\right) U_{[\zeta, \infty]}^{[n]}\right|_{\zeta^{n}=\eta^{n}, \zeta_{T}=\eta_{T}} .
\end{aligned}
$$

The subtractions at light-cone infinity are important to produce the missing transverse Wilson lines. It is shown in detail in ref. [9] how these subtractions $\left.A_{T}^{\alpha}(\eta)\right|_{\eta^{P}=\infty}$ from the 'higher twist' matrix elements with $A_{T}$ (and $A^{P}$ ) fields provide the missing transverse gauge connections $U_{\left[0_{T}, \xi T\right]}^{T}=U_{\left[0_{T}, \infty_{T}\right]}^{T} U_{\left[\infty_{T}, \xi_{T}\right]}^{T}$, which we will indicate as $U^{T}[p]$ with the argument $p$ again just indicating that the link connection involves the (transverse) endpoints 


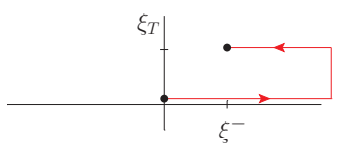

(a)

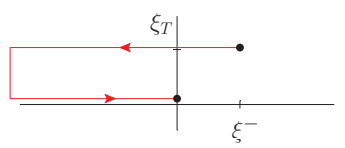

(b)

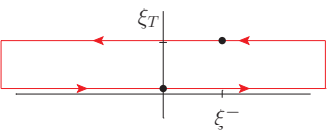

(c)

Figure 6. The gauge connections present in the expression for the unintegrated cross section in eq. (3.24), (a) $U_{+}^{\dagger} U_{+} \Rightarrow W_{+}^{[n]}=U_{[0, \infty]}^{[n]} U_{\left[0_{T}, \xi_{T}\right]}^{T} U_{[\infty, \xi]}^{[n]}$, (b) $U_{-}^{\dagger} U_{-} \Rightarrow W_{-}^{[n] \dagger}=$ $U_{[\xi,-\infty]}^{[n]} U_{\left[\xi, 0_{T}\right]}^{T} U_{[-\infty, 0]}^{[n]}$, and (c) $W_{\square}^{[n]}=W_{+}^{[n]} W_{-}^{[n] \dagger}$.

$0_{T}$ and $\xi_{T}$ conjugate to parton momentum $p$ and fields belonging to $\Phi(p)$. Incorporating all transverse gauge connections, we obtain (symmetrized) pieces

$$
U^{T}\left[p_{1}, p_{2}, k_{1}\right]=\mathcal{S}\left\{U^{T}\left[p_{1}\right] U^{T}\left[p_{2}\right] U^{T}\left[k_{1}\right]\right\}
$$

with irrelevant ordering among themselves. In the ordering of these pieces and the combination with collinear pieces of the gauge link, we will neglect contributions from commutators involving $A^{\mu}\left(p_{1}\right)$ and $A^{\nu}\left(p_{2}\right)$ since $\left(p_{1}-p_{2}\right)^{2} \sim Q^{2}$. It is clear that in kinematic regions where all parton momenta are small this cannot be used, complicating the full QCD analysis. Combined with the connections along the light-like direction, we now have for our tree-level analysis a set of entangled Wilson lines that bridge the non-locality of parton fields in the various correlators (see figure 5(b)). The resulting expression for the cross section is again of the form in equation eq. (3.12), but including transverse pieces, denoted $U_{+}[p, \ldots]=U^{T}[p, \ldots] U^{[n]}[p, \ldots]$, etc. One gets for the cross section an expression in terms of unintegrated correlators, which are only integrated over $p \cdot P$,

$$
\begin{aligned}
d \sigma & \sim \operatorname{Tr}_{c}\left[U_{-}^{\dagger}\left[p_{2}, k_{1}, k_{2}\right] \Phi\left(x_{1}, p_{1 T}\right) U_{-}\left[p_{2}, k_{1}, k_{2}\right] \Gamma_{1}^{*} U_{+}^{\dagger}\left[p_{1}, p_{2}, k_{2}\right] \Delta\left(z_{1}, k_{1 T}\right) U_{+}\left[p_{1}, p_{2}, k_{2}\right] \Gamma_{1}\right] \\
& \times \operatorname{Tr}_{c}\left[U_{-}^{\dagger}\left[p_{1}, k_{1}, k_{2}\right] \Phi\left(x_{2}, p_{2 T}\right) U_{-}\left[p_{1}, k_{1}, k_{2}\right] \Gamma_{2}^{*} U_{+}^{\dagger}\left[p_{1}, p_{2}, k_{1}\right] \Delta\left(z_{2}, k_{2 T}\right) U_{+}\left[p_{1}, p_{2}, k_{1}\right] \Gamma_{2}\right]
\end{aligned}
$$

This resulting expression is now color gauge-invariant. The Wilson lines can be taken along a generic $n$-direction, which even could be chosen different for each of the gauge connections in eq. (3.24), but its color structure is fully entangled and it does not allow for a factorized expression with universal correlators that have their own gauge links. Viewing it as a factorized expression it contains hard amplitudes, soft correlators and gauge connections, where the gauge connections take care of a 'color resetting' which feels all hadrons that are involved.

\section{Disentangling the color flow dependence}

Starting with the expression of eq. (3.24) for the cross section, we first look at the case that the hard process doesn't affect the color flow as in figure 2(a). This means that the vertices $\Gamma_{1}$ and $\Gamma_{2}$ are color independent. In our expression we have floating around in the full expression pieces, like $U^{T}[p] U_{+}^{[n]}[p] \ldots \Phi\left(x, p_{T}\right) \ldots U_{+}^{[n] \dagger}[p] U^{T \dagger}[p] \ldots$, which only when they are combined in a single trace would yield gauge links appropriate for TMDs,

$$
\Phi^{[ \pm]}\left(x, p_{T}\right)=\operatorname{Tr}_{c}\left[U^{T}[p] U_{+}^{[n]}[p] \Phi\left(x, p_{T}\right) U_{+}^{[n] \dagger}[p] U^{T \dagger}[p]\right]
$$




$$
\begin{aligned}
& =\left.\int \frac{d \xi \cdot P d^{2} \xi_{T}}{(2 \pi)^{3}} e^{i p \cdot \xi}\left\langle P\left|\bar{\psi}(0) U_{[0, \infty]}^{[n]} U_{\left[0_{T}, \xi_{T}\right]}^{T} U_{[\infty, \xi]}^{[n]} \psi(\xi)\right| P\right\rangle\right|_{L F} \\
& =\left.\int \frac{d \xi \cdot P d^{2} \xi_{T}}{(2 \pi)^{3}} e^{i p \cdot \xi}\left\langle P\left|\bar{\psi}(0) W_{ \pm[0, \xi]}^{[n]} \psi(\xi)\right| P\right\rangle\right|_{L F}=\operatorname{Tr}_{c}\left[W_{ \pm}^{[n]}[p] \Phi\left(x, p_{T}\right)\right],
\end{aligned}
$$

or

$$
\begin{aligned}
& \Delta^{[ \pm]}\left(z, k_{T}\right)=\frac{1}{N_{c}} \operatorname{Tr}_{c}\left[U^{T}[k] U_{ \pm}^{[n]}[k] \Delta\left(z, k_{T}\right) U_{ \pm}^{[n] \dagger}[k] U^{T \dagger}[k]\right]=\frac{1}{N_{c}} \operatorname{Tr}_{c}\left[\Delta\left(z, k_{T}\right) W_{ \pm}^{[n] \dagger}[k]\right] \\
& \quad=\left.\int \frac{d \xi \cdot K_{h} d^{2} \xi_{T}}{(2 \pi)^{3}} e^{-i k \cdot \xi} \frac{1}{N_{c}} \operatorname{Tr}_{c}\left\langle 0\left|U_{\left[\infty_{T}, 0_{T}\right]}^{T} U_{[ \pm \infty, 0]}^{[n]} \psi(\xi) a_{h}^{\dagger} a_{h} \bar{\psi}(0) U_{[\xi, \pm \infty]}^{[n]} U_{\left[\xi_{T}, \infty_{T}\right]}^{T}\right| 0\right\rangle\right|_{L F}
\end{aligned}
$$

with the coordinate space structure of $W_{ \pm[0, \xi]}^{[n]}$ shown in figures $6(\mathrm{a})$ and (b). Even if one is in the lucky situation that one can combine the relevant Wilson lines in a single trace, there still would be combinations left in other color traces which form Wilson loops $W_{\square}^{[n]}=W_{+}^{[n]} W_{-}^{[n] \dagger}$ as illustrated in figure $6(\mathrm{c})$.

\subsection{TMDs in 'elementary' processes}

Considering, as a reference, first the 'elementary' Drell-Yan (DY) process, which like other electroweak processes such as leptoproduction, or electron-positron annihilation is relatively simple, because the color flow through initial and final states consists of a single color loop. In that case, taking the DY process as an example (figure 7), the cross section is given by

$$
\begin{aligned}
d \sigma_{D Y} & \sim \operatorname{Tr}_{c}\left[U_{-}^{[n] \dagger}\left[p_{2}\right] \Phi\left(x_{1}, p_{1 T}\right) U_{-}^{[n]}\left[p_{2}\right] \Gamma^{*} U_{-}^{[n] \dagger}\left[p_{1}\right] \bar{\Phi}\left(x_{2}, p_{2 T}\right) U_{-}^{[n]}\left[p_{1}\right] \Gamma\right] \\
& =\operatorname{Tr}_{c}\left[W_{-}^{[n] \dagger}\left[p_{2}\right] \Phi\left(x_{1}, p_{1 T}\right) \Gamma^{*} \bar{\Phi}\left(x_{2}, p_{2 T}\right) W_{-}^{[n]}\left[p_{1}\right] \Gamma\right] .
\end{aligned}
$$

The gauge connections contain collinear and transverse fields and one has to be careful in disentangling. We note in particular that in general

$$
d \sigma_{D Y} \neq \operatorname{Tr}_{c}\left[W_{-}^{[n]}\left[p_{1}\right] \Phi\left(x_{1}, p_{1 T}\right)\right] \operatorname{Tr}_{c}\left[\bar{\Phi}\left(x_{2}, p_{2 T}\right) W_{-}^{[n] \dagger}\left[p_{2}\right]\right] \frac{1}{N_{c}} \Gamma \Gamma^{*} .
$$

However, if one of the hadrons can be treated collinear, i.e. integrating over one of the transverse momenta, say $p_{2 T}$, we can disentangle the traces and obtain

$$
\begin{aligned}
\frac{d \sigma_{D Y}}{d^{2} p_{1 T}} & \sim \operatorname{Tr}_{c}\left[W_{-}^{[n]}\left[p_{1}\right] \Phi\left(x_{1}, p_{1 T}\right)\right] \bar{\Phi}^{[W]}\left(x_{2}\right) \underbrace{\frac{1}{N_{c}} \Gamma \Gamma^{*}}_{\hat{\Sigma}_{D Y}} \\
& =\Phi^{[-]}\left(x_{1}, p_{1 T}\right) \Phi\left(x_{2}\right) \hat{\Sigma}_{D Y},
\end{aligned}
$$

where $\Phi^{[-]}\left(p_{1}\right)=\operatorname{Tr}_{c}\left[W_{-}^{[n]}\left[p_{1}\right] \Phi\left(p_{1}\right)\right]$. This illustrates the TMD factorization at tree-level and the relevant gauge links to be used, which was considered in detail in ref. [35]. Beyond tree-level one has the factorization formalism of Collins, Soper and Sterman [36], which has proven to be very successful for many applications, but does not catch all subtleties at small transverse momenta [18]. We will return to the problems with finding a full tree-level factorization in two TMDs in section 5 . 


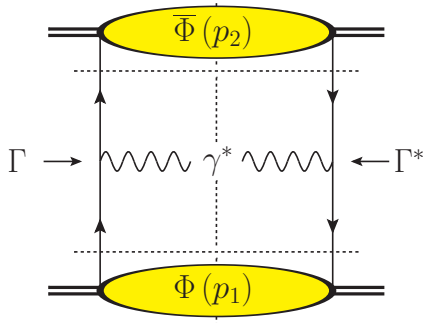

(a)

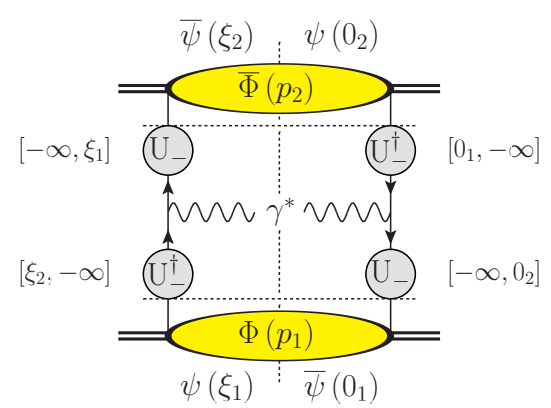

(b)

Figure 7. (a) The color-flow in the Drell-Yan process and (b) the gauge connections including their coordinate space structure.

\subsection{TMDs in 1-parton unintegrated processes}

In this subsection, we will turn to the situation of a more complicated color flow and we will show that TMDs with non-trivial gauge links appear in 1-parton unintegrated (1PU) contributions to the cross section, by which we mean contributions in which one has integrated over the parton transverse momenta in all but one of the hadrons. At this point we do not worry about experimental feasibility of measuring such a cross section. First assume as an intermediate step that only the transverse momentum of correlators $\Phi\left(p_{1}\right)$ and $\Phi\left(p_{2}\right)$ are left unintegrated, i.e. we look at jet production or non-hadronic final states. In that case one integrates over $k_{1 T}$ and $k_{2 T}$ and the collinear correlators $\Delta\left(z_{1}\right)$ and $\Delta\left(z_{2}\right)$ just involve unique collinear gauge links, while $W_{\square}\left[k_{1}\right]$ and $W_{\square}\left[k_{2}\right]$ become unity (in color space). The result is an expression of the form

$$
\begin{aligned}
d \sigma_{1} \sim & \operatorname{Tr}_{c}\left[U_{-}^{\dagger}\left[p_{2}\right] \Phi\left(x_{1}, p_{1 T}\right) U_{-}\left[p_{2}\right] \Gamma_{1}^{*} U_{+}^{\dagger}\left[p_{1}, p_{2}\right] \Delta^{[W]}\left(z_{1}\right) U_{+}\left[p_{1}, p_{2}\right] \Gamma_{1}\right] \\
& \times \operatorname{Tr}_{c}\left[U_{-}^{\dagger}\left[p_{1}\right] \Phi\left(x_{2}, p_{2 T}\right) U_{-}\left[p_{1}\right] \Gamma_{2}^{*} U_{+}^{\dagger}\left[p_{1}, p_{2}\right] \Delta^{[W]}\left(z_{2}\right) U_{+}\left[p_{1}, p_{2}\right] \Gamma_{2}\right],
\end{aligned}
$$

which is still entangled. This was the example discussed in ref. [37].

Integrating over $p_{2 T}$, the result reduces to

$$
\begin{aligned}
d \sigma_{1} \sim & \operatorname{Tr}_{c}\left[\Phi\left(x_{1}, p_{1 T}\right) \Gamma_{1}^{*} U_{+}^{\dagger}\left[p_{1}\right] \Delta^{[W]}\left(z_{1}\right) U_{+}\left[p_{1}\right] \Gamma_{1}\right] \\
& \times \operatorname{Tr}_{c}\left[U_{-}^{\dagger}\left[p_{1}\right] \Phi^{[W]}\left(x_{2}\right) U_{-}\left[p_{1}\right] \Gamma_{2}^{*} U_{+}^{\dagger}\left[p_{1}\right] \Delta^{[W]}\left(z_{2}\right) U_{+}\left[p_{1}\right] \Gamma_{2}\right] . \\
\sim & \operatorname{Tr}_{c}\left[\Phi\left(x_{1}, p_{1 T}\right) \Gamma_{1}^{*} \Delta^{[W]}\left(z_{1}\right) W_{+}\left[p_{1}\right] \Gamma_{1}\right] \operatorname{Tr}_{c}\left[W_{-}^{\dagger}\left[p_{1}\right] \Phi^{[W]}\left(x_{2}\right) \Gamma_{2}^{*} \Delta^{[W]}\left(z_{2}\right) W_{+}\left[p_{1}\right] \Gamma_{2}\right] . \\
= & \operatorname{Tr}_{c}\left[W_{+}^{[n]}\left[p_{1}\right] \Phi\left(x_{1}, p_{1 T}\right) \Gamma_{1}^{*} \Delta\left(z_{1}\right) \Gamma_{1}\right] \operatorname{Tr}_{c}\left[W_{\square}^{[n]}\left[p_{1}\right] \Phi\left(x_{2}\right) \Gamma_{2}^{*} \Delta\left(z_{2}\right) \Gamma_{2}\right]
\end{aligned}
$$

and gives

$$
\begin{aligned}
d \sigma_{1} & \sim \operatorname{Tr}_{c}\left[\Phi\left(x_{1}, p_{1 T}\right) W_{+}^{[n]}\left[p_{1}\right] \Gamma_{1}^{*} \Delta\left(z_{1}\right) \Gamma_{1}\right] \frac{1}{N_{c}} \operatorname{Tr}_{c}\left[W_{\square}^{[n]}\left[p_{1}\right]\right] \operatorname{Tr}_{c}\left[\Phi\left(x_{2}\right) \Gamma_{2}^{*} \Delta\left(z_{2}\right) \Gamma_{2}\right] \\
& =\Phi^{[+(\square)]}\left(x_{1}, p_{1 T}\right) \Phi\left(x_{2}\right) \underbrace{\Gamma_{1} \Gamma_{1}^{*} \Gamma_{2} \Gamma_{2}^{*}}_{\hat{\Sigma}_{1}} \Delta\left(z_{1}\right) \Delta\left(z_{2}\right)
\end{aligned}
$$

where Wilson lines combined with the correlators can be combined into TMD correlators with gauge links, such as $\Phi^{[+]}\left(p_{1}\right)=\operatorname{Tr}_{c}\left[\Phi\left(p_{1}\right) W_{+}^{[n]}\left[p_{1}\right]\right]$. In the step from (4.8) 


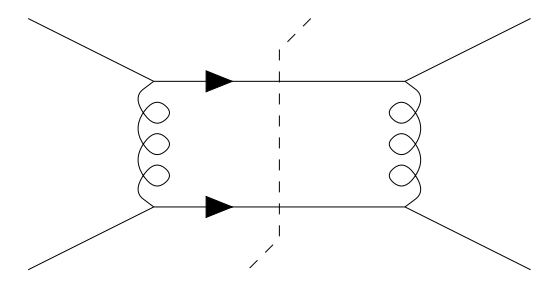

Figure 8. The squared amplitude for the hard interaction between two quarks coming from a one-gluon-exchange diagram. We consider for simplicity two quarks with different flavors.

to (4.9) we have split the second trace into two parts containing the trace of $W_{\square}^{[n]}$ (appropriately averaged) and the trace of the ( $n$-independent) collinear part. In the last step the trace of the Wilson loop (non-local in the coordinate $\xi$ conjugate to the parton momentum $p_{1}$ ) has been absorbed into a more complicated gauge link for $\Phi^{[+(\square)]}\left(x_{1}, p_{1 T}\right)=$ $\frac{1}{N_{c}} \operatorname{Tr}_{c}\left[W_{\square}^{[n]}\left[p_{1}\right]\right] \operatorname{Tr}_{c}\left[\Phi\left(p_{1}\right) W_{+}^{[n]}\left[p_{1}\right]\right]$. Note that, in spite of what the notation might suggest, $\Phi^{[+(\square)]}$ is not a multiplicative factor times $\Phi^{[+]}$because the fields in both factors belong to the same matrix element (indicated through argument $\left[p_{1}\right]$ ). These complex gauge links are the process-dependent gauge links discussed in refs. [10-12, 38, 39].

As a further illustration, we include the color flow possibilities in the case of a hard process where a gluon is exchanged in the hard part (see figure 8) instead of a colorless boson as in figure 2. One gets different color flow patterns that contribute giving rise to two different color contractions

$$
\begin{aligned}
d \sigma \sim & \operatorname{Tr}_{c}\left[U_{-}^{[n] \dagger}\left[p_{2}, k_{1}, k_{2}\right] \Phi\left(p_{1}\right) U_{-}^{[n]}\left[p_{2}, k_{1}, k_{2}\right] \Gamma_{1}^{*} T^{a} U_{+}^{[n] \dagger}\left[p_{1}, p_{2}, k_{2}\right] \Delta\left(k_{1}\right) U_{+}^{[n]}\left[p_{1}, p_{2}, k_{2}\right] \Gamma_{2} T^{b}\right] \\
& \times \operatorname{Tr}_{c}\left[U_{-}^{[n] \dagger}\left[p_{1}, k_{1}, k_{2}\right] \Phi\left(p_{2}\right) U_{-}^{[n]}\left[p_{1}, k_{1}, k_{2}\right] \Gamma_{2}^{*} T^{a} U_{+}^{[n] \dagger}\left[p_{1}, p_{2}, k_{1}\right] \Delta\left(k_{2}\right) U_{+}^{[n]}\left[p_{1}, p_{2}, k_{1}\right] \Gamma_{1} T^{b}\right] \\
= & \frac{N_{c}^{2}+1}{N_{c}^{2}-1} \operatorname{Tr}_{c}\left[U_{-}^{[n] \dagger}\left[p_{2}, k_{1}, k_{2}\right] \Phi\left(p_{1}\right) U_{-}^{[n]}\left[p_{2}, k_{1}, k_{2}\right] \Gamma_{1}^{*} U_{+}^{[n] \dagger}\left[p_{1}, p_{2}, k_{2}\right] \Delta\left(k_{1}\right) U_{+}^{[n]}\left[p_{1}, p_{2}, k_{2}\right] \Gamma_{2}\right] \\
& \quad \times \operatorname{Tr}_{c}\left[U_{-}^{[n] \dagger}\left[p_{1}, k_{1}, k_{2}\right] \Phi\left(p_{2}\right) U_{-}^{[n]}\left[p_{1}, k_{1}, k_{2}\right] \Gamma_{2}^{*} U_{+}^{[n] \dagger}\left[p_{1}, p_{2}, k_{1}\right] \Delta\left(k_{2}\right) U_{+}^{[n]}\left[p_{1}, p_{2}, k_{1}\right] \Gamma_{1}\right] \\
& -\frac{2}{N_{c}^{2}-1} \operatorname{Tr}_{c}\left[U_{-}^{[n] \dagger}\left[p_{2}, k_{1}, k_{2}\right] \Phi\left(p_{1}\right) U_{-}^{[n]}\left[p_{2}, k_{1}, k_{2}\right] \Gamma_{1}^{*} U_{+}^{[n] \dagger}\left[p_{1}, p_{2}, k_{2}\right] \Delta\left(k_{1}\right) U_{+}^{[n]}\left[p_{1}, p_{2}, k_{2}\right]\right. \\
& \left.\quad \times U_{-}^{[n] \dagger}\left[p_{1}, k_{1}, k_{2}\right] \Phi\left(p_{2}\right) U_{-}^{[n]}\left[p_{1}, k_{1}, k_{2}\right] \Gamma_{2}^{*} U_{+}^{[n] \dagger}\left[p_{1}, p_{2}, k_{1}\right] \Delta\left(k_{2}\right) U_{+}^{[n]}\left[p_{1}, p_{2}, k_{1}\right] \Gamma_{1}\right] \\
= & \frac{N_{c}^{2}+1}{N_{c}^{2}-1} d \sigma_{1}-\frac{2}{N_{c}^{2}-1} d \sigma_{2},
\end{aligned}
$$

normalized by the usual color factor, such that the coefficients in front of these terms add to one. The first part is the one considered above. For the second part, one gets upon integration over $k_{1 T}$ and $k_{2 T}$

$$
\begin{aligned}
d \sigma_{2} \sim \operatorname{Tr}_{c}[ & U_{-}^{[n] \dagger}\left[p_{2}\right] \Phi\left(x_{1}, p_{1 T}\right) U_{-}^{[n]}\left[p_{2}\right] \Gamma_{1}^{*} U_{+}^{[n] \dagger}\left[p_{1}, p_{2}\right] \Delta\left(z_{1}\right) U_{+}^{[n]}\left[p_{1}, p_{2}\right] \Gamma_{2} \\
& \left.\times U_{-}^{[n] \dagger}\left[p_{1}\right] \Phi\left(x_{2}, p_{2 T}\right) U_{-}^{[n]}\left[p_{1}\right] \Gamma_{2}^{*} U_{+}^{[n] \dagger}\left[p_{1}, p_{2}\right] \Delta\left(z_{2}\right) U_{+}^{[n]}\left[p_{1}, p_{2}\right] \Gamma_{1}\right]
\end{aligned}
$$

leaving an entangled situation. If only one transverse momentum remains unintegrated, one again can combine the result into a single complicated gauge link,

$$
\begin{aligned}
d \sigma_{2} \sim \operatorname{Tr}_{c}\left[\Phi\left(x_{1}, p_{1 T}\right) \Gamma_{1}^{*} U_{+}^{[n] \dagger}\left[p_{1}\right] \Delta\left(z_{1}\right) U_{+}^{[n]}\left[p_{1}\right] \Gamma_{2} U_{-}^{[n] \dagger}\left[p_{1}\right]\right. \\
\left.\times \Phi\left(x_{2}\right) U_{-}^{[n]}\left[p_{1}\right] \Gamma_{2}^{*} U_{+}^{[n] \dagger}\left[p_{1}\right] \Delta\left(z_{2}\right) U_{+}^{[n]}\left[p_{1}\right] \Gamma_{1}\right]
\end{aligned}
$$




$$
\begin{aligned}
& =\operatorname{Tr}_{c}\left[\Phi\left(x_{1}, p_{1 T}\right) W_{+}^{[n]}\left[p_{1}\right] \Gamma_{1}^{*} \Delta\left(z_{1}\right) \Gamma_{2} \Phi\left(x_{2}\right) W_{\square}^{[n]}\left[p_{1}\right] \Gamma_{2}^{*} \Delta\left(z_{2}\right) \Gamma_{1}\right] \\
& =\Phi^{[+\square]}\left(x_{1}, p_{1 T}\right) \Phi\left(x_{2}\right) \underbrace{\Gamma_{1} \Gamma_{1}^{*} \Gamma_{2} \Gamma_{2}^{*}}_{\hat{\Sigma}_{2}} \Delta\left(z_{1}\right) \Delta\left(z_{2}\right),
\end{aligned}
$$

where $\Phi^{[+\square]}\left(p_{1}\right)=\operatorname{Tr}_{c}\left[\Phi\left(p_{1}\right) W_{+}^{[n]}\left[p_{1}\right] W_{\square}^{[n]}\left[p_{1}\right]\right]$. Combining both contributions one gets the result [11],

$$
d \sigma \sim\left(\frac{N_{c}^{2}+1}{N_{c}^{2}-1} \Phi^{[+(\square)]}\left(x_{1}, p_{1 T}\right) \Phi\left(x_{2}\right) \hat{\Sigma}_{1}-\frac{2}{N_{c}^{2}-1} \Phi^{[+\square]}\left(x_{1}, p_{1 T}\right) \Phi\left(x_{2}\right) \hat{\Sigma}_{2}\right) \Delta\left(z_{1}\right) \Delta\left(z_{2}\right) .
$$

In this particular case $\hat{\Sigma}_{1}$ and $\hat{\Sigma}_{2}$ are the same, but in general one has more diagrams contributing to the hard process, each contribution gets split up into its own color patterns. The results for such 1PU processes have been tabulated in ref. [11].

\section{Analysis in terms of transverse moments}

In this section, we will start with the unintegrated result in eq. (3.24) and take transverse moments, which will give collinear results, which include correlators with covariant derivatives $i D_{T}^{\alpha}$ and $A_{T}^{\alpha}$ or in gauge-invariant form integrals over $G^{n \alpha}$. The transverse moments are obtained by looking at weighted cross sections of the form

$$
\left\langle p_{T}^{\alpha_{1}} \ldots p_{T}^{\alpha_{N}} \sigma\right\rangle=\int d^{2} p_{T} p_{T}^{\alpha_{1}} \ldots p_{T}^{\alpha_{N}} \frac{d \sigma}{d^{2} p_{T}} .
$$

We will apply this to the cross section $\sigma$, built from the two pieces $\sigma_{1}$ and $\sigma_{2}$, discussed in the previous paragraph. The simplest, lowest, transverse moment is the integrated cross section,

$$
\langle\sigma\rangle=\int d^{2} p_{T} \frac{d \sigma}{d^{2} p_{T}}=\Phi\left(x_{1}\right) \Phi\left(x_{2}\right) \underbrace{\left(\frac{N_{c}^{2}+1}{N_{c}^{2}-1} \hat{\Sigma}_{1}-\frac{2}{N_{c}^{2}-1} \hat{\Sigma}_{2}\right)}_{\hat{\Sigma}} \Delta\left(z_{1}\right) \Delta\left(z_{2}\right),
$$

which has between brackets the standard partonic cross section $\hat{\Sigma}$.

\subsection{Single weighted asymmetries}

To see what happens with the first transverse moment, we need to investigate at the level of correlators what happens when one applies $i \partial_{\xi}^{\alpha}$ to the matrix elements. To clarify the role of the gauge link in a correlator, in particular the contribution at infinity, we consider

$$
i \partial_{T}^{\alpha}\left[\ldots U_{[0, \infty]}^{[n]} U_{\left[0_{T}, \xi_{T}\right]}^{T} U_{[\infty, \xi]}^{[n]} \ldots \psi(\xi)\right]=\left[\ldots U_{[0, \infty]}^{[n]} U_{\left[0_{T}, \xi_{T}\right]}^{T} i D_{T}^{\alpha} U_{[\infty, \xi]}^{[n]} \ldots \psi(\xi)\right] .
$$

We can evaluate explicitly

$$
i D_{T}^{\alpha} U_{[\infty, \xi]}^{[n]} \ldots \psi(\xi)=U_{[\infty, \xi]}^{[n]} i D_{T}^{\alpha}(\xi) \ldots \psi(\xi)-\int_{\infty}^{\xi} d \eta \cdot P U_{[\infty, \eta]}^{[n]} G_{T}^{n \alpha}(\eta) U_{[\eta, \xi]}^{[n]} \ldots \psi(\xi),
$$

noting that the second term depends through the integration limits on the structure of the Wilson line, in particular on the transverse piece being at plus or minus infinity. We could 
simply use the second term as a definition of the $A_{T}^{\alpha}$ field but such a definition would for instance not have the correct time-reversal property. We will use a time-reversal-definite expression for $A^{\alpha}(\xi)$,

$$
A^{\alpha}(\xi) \equiv \int_{-\infty}^{\infty} d \eta \cdot P \epsilon\left(\xi^{P}-\eta^{P}\right) U_{[\xi, \eta]}^{[n]} G^{n \alpha}(\eta) U_{[\eta, \xi]}^{[n]},
$$

which is gauge-equivalent to the expressions in eqs. (3.21) and (3.22). The form in eq. (5.5) implicitly implies $A^{n}=0$ and antisymmetric boundary conditions at $\xi^{P}= \pm \infty$ for $A_{T}^{\alpha}(\xi)$ and $A^{P}(\xi)$,

$$
U_{[\xi, \infty]}^{[n]} A^{\alpha}\left(\infty, \xi_{T}, \xi^{n}\right) U_{[\infty, \xi]}^{[n]}+U_{[\xi,-\infty]}^{[n]} A^{\alpha}\left(-\infty, \xi_{T}, \xi^{n}\right) U_{[-\infty, \xi]}^{[n]}=0,
$$

while

$$
\begin{gathered}
U_{[\xi, \infty]}^{[n]} A^{\alpha}\left(\infty, \xi_{T}, \xi^{n}\right) U_{[\infty, \xi]}^{[n]}-U_{[\xi,-\infty]}^{[n]} A^{\alpha}\left(-\infty, \xi_{T}, \xi^{n}\right) U_{[-\infty, \xi]}^{[n]} \\
=\int_{-\infty}^{\infty} d \xi \cdot P G^{n \alpha}(\xi)=2 \pi \widetilde{G}^{n \alpha}\left(\xi_{T}, \xi^{n}\right),
\end{gathered}
$$

or combining these two equations,

$$
U_{[\xi, \infty]}^{[n]} A^{\alpha}\left(\infty, \xi_{T}, \xi^{n}\right) U_{[\infty, \xi]}^{[n]}=-U_{[\xi,-\infty]}^{[n]} A^{\alpha}\left(-\infty, \xi_{T}, \xi^{n}\right) U_{[-\infty, \xi]}^{[n]}=\pi \widetilde{G}^{n \alpha}\left(\xi_{T}, \xi^{n}\right) .
$$

The integral expression for $\pi \widetilde{G}^{n \alpha}$ represents actually the Fourier transform of the field strength tensor at zero momentum in the $n$-component (which is indicated by including a tilde over the $G$ and omitting $\xi^{P}$ from the argument list). Eq. (5.4) becomes

$$
i D_{T}^{\alpha} U_{[\infty, \xi]}^{[n]} \ldots \psi(\xi)=U_{[\infty, \xi]}^{[n]}\left(i D_{T}^{\alpha}(\xi)-A_{T}^{\alpha}(\xi)+\pi \widetilde{G}^{n \alpha}(\xi)\right) \ldots \psi(\xi) .
$$

To implement this in correlators, we consider besides the TMD correlator $\Phi\left(x, p_{T}\right)$ in eq. (4.1) the collinear matrix elements $\Phi_{A}^{\alpha}\left(x, x_{1}\right)$, which are the integrated quark-quarkgluon correlators starting with eq. (2.2). Including Wilson lines, this collinear correlator is of the form

$$
\Phi_{A}^{\alpha}\left(x, x_{1}\right)=\left.\int \frac{d \xi \cdot P}{2 \pi} \frac{d \eta \cdot P}{2 \pi} e^{i\left(p-p_{1}\right) \cdot \xi} e^{i p_{1} \cdot \eta}\left\langle P\left|\bar{\psi}(0) U_{[0, \eta]}^{[n]} A^{\alpha}(\eta) U_{[\eta, \xi]}^{[n]} \psi(\xi)\right| P\right\rangle\right|_{L C},
$$

or integrated over $x_{1}$,

$$
\Phi_{A}^{\alpha}(x)=\int d x_{1} \Phi_{A}^{\alpha}\left(x, x_{1}\right)=\left.\int \frac{d \xi \cdot P}{2 \pi} e^{i p \cdot \xi}\left\langle P\left|\bar{\psi}(0) U_{[0, \xi]}^{[n]} A^{\alpha}(\xi) \psi(\xi)\right| P\right\rangle\right|_{L C} .
$$

Similarly we define $\Phi_{D}^{\alpha}\left(x, x_{1}\right)$ and $\Phi_{G}^{\alpha}\left(x, x_{1}\right)$ using $i D^{\alpha}(\eta)$ and $G^{n \alpha}(\eta)$, respectively. The latter is given by

$$
\Phi_{G}^{\alpha}\left(x, x_{1}\right)=\left.\int \frac{d \xi \cdot P}{2 \pi} \frac{d \eta \cdot P}{2 \pi} e^{i\left(p-p_{1}\right) \cdot \xi} e^{i p_{1} \cdot \eta}\left\langle P\left|\bar{\psi}(0) U_{[0, \eta]}^{[n]} G^{n \alpha}(\eta) U_{[\eta, \xi]}^{[n]} \psi(\xi)\right| P\right\rangle\right|_{L C} .
$$


The relation in eq. (5.5) implies for these correlators

$$
\Phi_{A}^{\alpha}(x)=\mathrm{PV} \int d x_{1} \frac{i}{x-x_{1}} \Phi_{G}^{\alpha}\left(x, x_{1}\right) .
$$

The operator $\widetilde{G}^{n \alpha}\left(\xi_{T}, \xi^{n}\right)$ in eq. (5.7) appears in the correlator with a gluon field strength, but at zero $n$-component of the momentum, i.e. at $x_{1}=0$. The other (among them transverse) components of the gluon momentum are also integrated over in eq. (5.12). This particular correlator, referred to as a gluonic pole matrix element,

$$
\Phi_{G}^{\alpha}(x, x)=\left.\int \frac{d \xi \cdot P}{2 \pi} e^{i p \cdot \xi}\left\langle P\left|\bar{\psi}(0) U_{[0, \xi]}^{[n]} \widetilde{G}^{n \alpha} \psi(\xi)\right| P\right\rangle\right|_{L C}
$$

will play an important role for transverse moments. Note that we have not included any argument for the field strenght in $\left.\widetilde{G}^{n \alpha} \psi(x)\right|_{L C}$, because all arguments become zero after integration over $p_{1 T}, p_{1} \cdot P$ (implying $\xi_{T}=\eta_{T}$ and $\xi^{n}=\eta^{n}$ ) followed by the integration over $p_{T}$ and $p \cdot P$ (putting $\xi$ on the light-cone, i.e. $\xi_{T}=0_{T}$ and $\xi^{n}=0$ ).

Using the expressions in eqs. (5.3) and (5.4), we consider the following unintegrated TMD functions starting with $\Phi^{[ \pm]}\left(x, p_{T}\right)$,

$$
\begin{aligned}
\Phi_{\partial}^{\alpha[ \pm]}\left(x, p_{T}\right) & \equiv p_{T}^{\alpha} \Phi^{[ \pm]}\left(x, p_{T}\right) \\
& =\left.\int \frac{d \xi \cdot P d^{2} \xi_{T}}{(2 \pi)^{3}} e^{i p \cdot \xi}\left\langle P\left|\bar{\psi}(0) U_{[0, \pm \infty]}^{[n]} U_{\left[0_{T}, \xi_{T}\right]}^{T} i D_{T}^{\alpha} U_{[ \pm \infty, \xi]}^{[n]} \psi(\xi)\right| P\right\rangle\right|_{L F} .
\end{aligned}
$$

Using the result in eq. (5.9) one gets

$$
\Phi_{\partial}^{\alpha[ \pm]}\left(x, p_{T}\right)=\widetilde{\Phi}_{\partial}^{\alpha[ \pm]}\left(x, p_{T}\right) \pm \pi \Phi_{G}^{\alpha[ \pm]}\left(x, p_{T}\right),
$$

where

$$
\begin{aligned}
\pi \Phi_{G}^{\alpha[ \pm]}\left(x, p_{T}\right) & = \pm\left.\int \frac{d \xi \cdot P d^{2} \xi_{T}}{(2 \pi)^{3}} e^{i p \cdot \xi}\left\langle P\left|\bar{\psi}(0) U_{[0, \pm \infty]}^{[n]} U_{\left[0_{T}, \xi_{T}\right]}^{T} A_{T}^{\alpha}\left( \pm \infty, \xi_{T}\right) U_{[ \pm \infty, \xi]}^{[n]} \psi(\xi)\right| P\right\rangle\right|_{L F} \\
& =\left.\int \frac{d \xi \cdot P d^{2} \xi_{T}}{(2 \pi)^{3}} e^{i p \cdot \xi}\left\langle P\left|\bar{\psi}(0) W_{ \pm[0, \xi]}^{[n]} \pi \widetilde{G}_{T}^{n \alpha}\left(\xi_{T}\right) \psi(\xi)\right| P\right\rangle\right|_{L F} \\
\widetilde{\Phi}_{\partial}^{\alpha[ \pm]}\left(x, p_{T}\right) & =\left.\int \frac{d \xi \cdot P d^{2} \xi_{T}}{(2 \pi)^{3}} e^{i p \cdot \xi}\left\langle P\left|\bar{\psi}(0) W_{ \pm[0, \xi]}^{[n]} i \partial_{T}^{\alpha} \psi(\xi)\right| P\right\rangle\right|_{L F} \\
& =\left.\int \frac{d \xi \cdot P d^{2} \xi_{T}}{(2 \pi)^{3}} e^{i p \cdot \xi}\left\langle P\left|\bar{\psi}(0) W_{ \pm[0, \xi]}^{[n]}\left(i D_{T}^{\alpha}(\xi)-A_{T}^{\alpha}(\xi)\right) \psi(\xi)\right| P\right\rangle\right|_{L F}
\end{aligned}
$$

or after integration over $p_{T}$,

$$
\begin{aligned}
\Phi_{\partial}^{\alpha}{ }^{[ \pm]}(x) & \equiv \int d^{2} p_{T} p_{T}^{\alpha} \Phi^{[ \pm]}\left(x, p_{T}\right)=\Phi_{D}^{\alpha}(x)-\int d x_{1} \frac{i}{x-x_{1} \mp i \epsilon} \Phi_{G}^{\alpha}\left(x, x_{1}\right) \\
& =\underbrace{\Phi_{D}^{\alpha}(x)-\Phi_{A}^{\alpha}(x)}_{\widetilde{\Phi}_{\partial}^{\alpha}(x)} \pm \pi \Phi_{G}^{\alpha}(x, x) .
\end{aligned}
$$

We note that the sole dependence on the direction of the gauge link is in the sign in front of the gluonic pole correlator. 
In the analysis presented here, we have tacitly assumed that the Wilson line in combination with the quark fields could be combined into a correlator with a gauge link and we only considered the cases of the simplest TMDs $\Phi^{[ \pm]}$(without Wilson loops). To see what is happening in general we must realize that the complications arise from differentiating the Wilson line and obtaining covariant derivatives (in the above expressions the $i D_{T}^{\alpha}$ ) in $\Phi_{\partial}^{\alpha}$. This result is then separated into two parts, the parts $\widetilde{\Phi}_{\partial}^{\alpha}$, which has the same color structure as $\Phi$ itself and a gluonic pole part of which the color structure is $\Phi_{G}^{\alpha}(x, x)=\operatorname{Tr}_{c}\left[\Phi_{8}(x) \tilde{G}^{n \alpha}[p]\right]$, where the argument $[p]$ of $\widetilde{G}^{n \alpha}$ (see eq. (5.14)) just indicates its (spatial) connection to the coordinates in quark-quark correlator $\Phi(p)$, similarly as has been used for the Wilson lines $U[p]$. In the entangled expressions for the cross sections we have to use

$$
\begin{aligned}
\left.i \partial_{1 T}^{\alpha}\left(\Phi\left(p_{1}\right) \ldots U_{-}^{[n] \dagger}\left[p_{1}\right] \Phi\left(p_{2}\right) U_{-}^{[n]}\left[p_{1}\right] \ldots\right)\right|_{L C} \\
\quad=\left(\widetilde{\Phi}_{\partial}^{\alpha}\left(p_{1}\right) \ldots \Phi\left(p_{2}\right) \ldots\right)+\left(\Phi_{8}\left(x_{1}\right) \ldots \pi \widetilde{G}^{n \alpha}\left[p_{1}\right] \Phi\left(p_{2}\right) \ldots\right) .
\end{aligned}
$$

After further color disentangling of the right-hand-side, where one must be careful because $\Phi\left(p_{2}\right)$ and possible other entries can have an octet structure, one then can recombine parts into $\pi \Phi_{G}^{\alpha}(x, x)=\operatorname{Tr}_{c}\left[\Phi_{8}(x) \pi \widetilde{G}^{n \alpha}[p]\right]$, as explained for a full quark-quark-gluon correlator following eq. (2.2). For an antiquark correlator $\bar{\Phi}\left(p_{2}\right)$ one obtains

$$
\begin{aligned}
\left.i \partial_{1 T}^{\alpha}\left(\Phi\left(p_{1}\right) \ldots U_{-}^{[n] \dagger}\left[p_{1}\right] \bar{\Phi}_{1}\left(p_{2}\right) U_{-}^{[n]}\left[p_{1}\right] \ldots\right)\right|_{L C} \\
\quad=\left(\widetilde{\Phi}_{\partial}^{\alpha}\left(p_{1}\right) \ldots \bar{\Phi}\left(p_{2}\right) \ldots\right)-\left(\Phi_{8}\left(x_{1}\right) \ldots \bar{\Phi}\left(p_{2}\right) \pi \widetilde{G}^{n \alpha}\left[p_{1}\right] \ldots\right) .
\end{aligned}
$$

Starting with the cross section $\sigma_{1}$ in eq. (4.8) we find that weighting with transverse momentum gives

$$
\begin{aligned}
\left\langle p_{1 T}^{\alpha} \sigma_{1}\right\rangle \sim & \operatorname{Tr}_{c}\left[\widetilde{\Phi}_{\partial}^{\alpha}\left(x_{1}\right) \Gamma_{1}^{*} \Delta\left(z_{1}\right) \Gamma_{1}\right] \operatorname{Tr}_{c}\left[\Phi\left(x_{2}\right) \Gamma_{2}^{*} \Delta\left(z_{2}\right) \Gamma_{2}\right] \\
& +\operatorname{Tr}_{c}\left[\Phi_{8}\left(x_{1}\right) \pi \widetilde{G}^{n \alpha}\left[p_{1}\right] \Gamma_{1}^{*} \Delta\left(z_{1}\right) \Gamma_{1}\right] \operatorname{Tr}_{c}\left[\Phi\left(x_{2}\right) \Gamma_{2}^{*} \Delta\left(z_{2}\right) \Gamma_{2}\right] \\
& +\operatorname{Tr}_{c}\left[\Phi_{8}\left(x_{1}\right) \Gamma_{1}^{*} \Delta\left(z_{1}\right) \Gamma_{1}\right] \operatorname{Tr}_{c}\left[\Phi\left(x_{2}\right) 2 \pi \widetilde{G}^{n \alpha}\left[p_{1}\right] \Gamma_{2}^{*} \Delta\left(z_{2}\right) \Gamma_{2}\right] .
\end{aligned}
$$

The traces in the last term are zero and we are left with

$$
\left\langle p_{1 T}^{\alpha} \sigma_{1}\right\rangle \sim\left(\widetilde{\Phi}_{\partial}^{\alpha}\left(x_{1}\right)+\pi \Phi_{G}^{\alpha}\left(x_{1}, x_{1}\right)\right) \Phi\left(x_{2}\right) \hat{\Sigma}_{1} \Delta\left(z_{1}\right) \Delta\left(z_{2}\right) .
$$

The weighted cross section of eq. (4.13) gives

$$
\begin{aligned}
\left\langle p_{1 T}^{\alpha} \sigma_{2}\right\rangle \sim & \operatorname{Tr}_{c}\left[\widetilde{\Phi}_{\partial}^{\alpha}\left(x_{1}\right) \Gamma_{1}^{*} \Delta\left(z_{1}\right) \Gamma_{2} \Phi\left(x_{2}\right) \Gamma_{2}^{*} \Delta\left(z_{2}\right) \Gamma_{1}\right] \\
& +\operatorname{Tr}_{c}\left[\Phi_{8}\left(x_{1}\right) \pi \widetilde{G}^{n \alpha}\left[p_{1}\right] \Gamma_{1}^{*} \Delta\left(z_{1}\right) \Gamma_{2} \Phi\left(x_{2}\right) \Gamma_{2}^{*} \Delta\left(z_{2}\right) \Gamma_{1}\right] \\
& +\operatorname{Tr}_{c}\left[\Phi_{8}\left(x_{1}\right) \Gamma_{1}^{*} \Delta\left(z_{1}\right) \Gamma_{2} \Phi\left(x_{2}\right) 2 \pi \widetilde{G}^{n \alpha}\left[p_{1}\right] \Gamma_{2}^{*} \Delta\left(z_{2}\right) \Gamma_{1}\right] .
\end{aligned}
$$

All terms survive and we are left with

$$
\left\langle p_{1 T}^{\alpha} \sigma_{2}\right\rangle \sim\left(\widetilde{\Phi}_{\partial}^{\alpha}\left(x_{1}\right)+3 \pi \Phi_{G}^{\alpha}\left(x_{1}, x_{1}\right)\right) \Phi\left(x_{2}\right) \hat{\Sigma}_{2} \Delta\left(z_{1}\right) \Delta\left(z_{2}\right) .
$$

Combining both contributions in the same way as in eq. (4.15), one obtains the result [11],

$$
\left\langle p_{1 T}^{\alpha} \sigma\right\rangle \sim\left(\frac{N_{c}^{2}+1}{N_{c}^{2}-1} \Phi_{\partial}^{\alpha[+(\square)]}\left(x_{1}\right) \Phi\left(x_{2}\right) \hat{\Sigma}_{1}-\frac{2}{N_{c}^{2}-1} \Phi_{\partial}^{\alpha[+\square]}\left(x_{1}\right) \Phi\left(x_{2}\right) \hat{\Sigma}_{2}\right) \Delta\left(z_{1}\right) \Delta\left(z_{2}\right)
$$




$$
\begin{aligned}
= & \widetilde{\Phi}_{\partial}^{\alpha}\left(x_{1}\right) \Phi\left(x_{2}\right) \underbrace{\left(\frac{N_{c}^{2}+1}{N_{c}^{2}-1} \hat{\Sigma}_{1}-\frac{2}{N_{c}^{2}-1} \hat{\Sigma}_{2}\right)}_{\hat{\Sigma}} \Delta\left(z_{1}\right) \Delta\left(z_{2}\right) \\
& +\pi \Phi_{G}^{\alpha}\left(x_{1}, x_{1}\right) \Phi\left(x_{2}\right) \underbrace{\left(\frac{N_{c}^{2}+1}{N_{c}^{2}-1} \hat{\Sigma}_{1}-\frac{6}{N_{c}^{2}-1} \hat{\Sigma}_{2}\right)}_{\hat{\Sigma}_{G P}} \Delta\left(z_{1}\right) \Delta\left(z_{2}\right),
\end{aligned}
$$

where the combination of hard squared amplitudes in the second term of eq. (5.27) is referred to as the gluonic pole cross section. In the case of quark-quark scattering with distinguishable quarks $\left(\hat{\Sigma}_{1}=\hat{\Sigma}_{2}\right)$ one thus has $\hat{\Sigma}_{G P}=\frac{N_{c}^{2}-5}{N_{c}^{2}-1} \hat{\Sigma}$, and for $N_{c}=3$ we get

$$
\left\langle p_{1 T}^{\alpha} \sigma\right\rangle \sim\left(\widetilde{\Phi}_{\partial}^{\alpha}\left(x_{1}\right) \Phi\left(x_{2}\right) \hat{\Sigma}+\pi \Phi_{G}^{\alpha}\left(x_{1}, x_{1}\right) \Phi\left(x_{2}\right) \frac{1}{2} \hat{\Sigma}\right) \Delta\left(z_{1}\right) \Delta\left(z_{2}\right) .
$$

In this result the two pieces in the cross section experimentally can be distinguished because of their time-reversal behavior. For instance single spin asymmetries have opposite time reversal behavior as compared to spin-averaged or double spin asymmetries. This time reversal behavior affects the parametrization of the gluonic pole matrix element (containing T-odd distribution functions such as Sivers and Boer-Mulders functions). These functions appear convoluted with the gluonic pole cross section rather than the standard partonic cross section. For hadron-hadron scattering the partonic cross sections and gluonic pole cross sections have been tabulated in ref. [39].

At this point we want to comment on the usefulness of our diagrammatic approach starting with an assumed convolution of soft correlators $\Phi\left(x, p_{T}\right)$ (integrated over $p \cdot P$ ) and hard amplitudes. For this we have to realize that the operators involved have particular canonical dimensions $d$ and twist $t$. Assignment of definite twist is only possible for local matrix elements or collinear correlators, e.g. the quark-quark (or gluon-gluon) correlators $\Phi^{[t=2]}(x), \Phi^{[t=3]}(x), \ldots$ or quark-quark-gluon correlators $\Phi_{D}^{[t=3]}(x)$ and $\Phi_{A}^{[t=3]}(x)$. We have schematically

$$
\Phi^{[d=2]}\left(x, p_{T}\right)=\Phi^{[t \geq 2]}\left(x, p_{T}\right),
$$

indicating on the right-hand-side that operators are involved of arbitrary twist with the minimal twist being the canonical dimension.

Now, let us look at an observable at a high-energy scale $Q$ including its dependence on collinear fractions and transverse momenta assuming appropriate identification of parton variables with kinematical variables, which for transverse momenta in hadron-hadron scattering would be $q_{T}=p_{1 T}+p_{2 T}$. We have

$$
\begin{aligned}
d \sigma\left(x_{1}, x_{2}, q_{T} ; Q\right)= & \Phi^{[d=2]}\left(x_{1}, p_{1 T}\right) \otimes \Phi^{[d=2]}\left(x_{2}, p_{2 T}\right) \otimes \hat{\Sigma} \\
& +\frac{M}{Q}\left[\Phi^{[d=2]}\left(x_{1}, p_{1 T}\right) \otimes \Phi^{[d=2]}\left(x_{2}, p_{2 T}\right) \otimes \hat{\Sigma}\right] \\
& +\frac{1}{Q}\left[\Phi^{[d=3]}\left(x_{1}, p_{1 T}\right) \otimes \Phi^{[d=2]}\left(x_{2}, p_{2 T}\right) \otimes \hat{\Sigma}\right] \\
& +\frac{1}{Q}\left[\Phi^{[d=2]}\left(x_{1}, p_{1 T}\right) \otimes \Phi^{[d=3]}\left(x_{2}, p_{2 T}\right) \otimes \hat{\Sigma}\right] \\
& +\ldots
\end{aligned}
$$


of which only the first term survives at $Q \rightarrow \infty$. Looking at the $q_{T}$-averaged (i.e. integrated) cross section, we have as leading contribution

$$
\langle\sigma\rangle\left(x_{1}, x_{2} ; Q\right) \quad \stackrel{Q \rightarrow \infty}{\longrightarrow} \Phi^{[t=2]}\left(x_{1}\right) \otimes \Phi^{[t=2]}\left(x_{2}\right) \otimes \hat{\Sigma},
$$

which summarizes the collinear approach. The leading contribution in the $q_{T}$-weighted cross section is given by

$$
\begin{aligned}
&\left\langle q_{T} \sigma\right\rangle\left(x_{1}, x_{2} ; Q\right) \quad \stackrel{Q \rightarrow \infty}{\longrightarrow} \quad\left\langle p_{1 T} \Phi^{[d=2]}\right\rangle\left(x_{1}\right) \otimes \Phi^{[t=2]}\left(x_{2}\right) \otimes \hat{\Sigma} \\
&+\Phi^{[t=2]}\left(x_{1}\right) \otimes\left\langle p_{2 T} \Phi^{[d=2]}\right\rangle\left(x_{2}\right) \otimes \hat{\Sigma}
\end{aligned}
$$

where the weighted TMD correlators contain actually twist-three and higher twist operators, schematically

$$
\left\langle p_{T} \Phi^{[d=2]}\right\rangle(x)=\Phi^{[t \geq 3]}(x) .
$$

Among the twist three correlators we have T-even correlators $\Phi_{D}^{[t=3]}, \Phi_{A}^{[t=3]}$ in the combination $\widetilde{\Phi}_{\partial}^{[t=3]}$ and the T-odd gluonic pole correlator $\Phi_{G}^{[t=3]}(x, x)$. The usefulness of the diagrammatic approach starting with eq. (5.29) is in its ability to provide us in a straightforward way with the tree-level coefficients of the relevant (combinations of) twist-three collinear correlators through the intermediate step of TMD correlators with a complex (process-dependent) gauge link. Similar results for the weighted asymmetries can also be obtained using collinear functions including the gluonic pole or ETQS functions directly from the start [40, 41]. The tree-level results of the diagrammatic approach, of course, need to be improved upon by including NLO QCD contributions. In line with our proof of absorbing all gauge connections into a gauge link in 1PU processes, the diagrammatic approach also provides the tree-level results for higher transverse moments as long as only one initial state hadron is involved. This is useful first of all for leptoproduction processes involving a single incoming hadron and, furthermore, for selected asymmetries in hadron-hadron scattering.

\subsection{Double weighted asymmetries}

We want to use the analysis in terms of transverse moments to indicate what is happening when the transverse momentum of two hadrons is involved, e.g. a double Sivers or double Boer-Mulders effect. The weightings with $p_{1 T}^{\alpha} p_{1 T}^{\beta}$ or with $p_{2 T}^{\alpha} p_{2 T}^{\beta}$ can straightforwardly be obtained from eq. (4.15), although the splitting of TMDs with more complicated gauge links in T-even and T-odd parts is certainly not trivial. We will investigate the weighting with $p_{1 T}^{\alpha} p_{2 T}^{\beta}$ following the same steps as done in the above for the single moments.

We will first turn to an 'elementary' process, namely DY scattering. Having one color loop, one finds that in single and double weighting one needs

$$
\begin{aligned}
\operatorname{Tr}_{c}[I] & =\frac{1}{N_{c}} \operatorname{Tr}_{c}[I] \operatorname{Tr}_{c}[I], \\
\operatorname{Tr}_{c}\left[T^{a} T^{a}\right] & =\frac{1}{N_{c}} \operatorname{Tr}_{c}\left[T^{a} T^{a}\right] \operatorname{Tr}_{c}[I], \\
\operatorname{Tr}_{c}\left[T^{a} T^{b} T^{a} T^{b}\right] & =-\frac{1}{N_{c}\left(N_{c}^{2}-1\right)} \operatorname{Tr}_{c}\left[T^{a} T^{a}\right] \operatorname{Tr}_{c}\left[T^{b} T^{b}\right]
\end{aligned}
$$


yielding

$$
\begin{gathered}
\left\langle p_{1 T}^{\alpha} p_{2 T}^{\beta} \sigma_{D Y}\right\rangle=\left(\widetilde{\Phi}_{\partial}^{\alpha}\left(x_{1}\right) \widetilde{\bar{\Phi}}_{\partial}^{\beta}\left(x_{2}\right)-\pi \Phi_{G}^{\alpha}\left(x_{1}, x_{1}\right) \widetilde{\bar{\Phi}}_{\partial}^{\beta}\left(x_{2}\right)-\widetilde{\Phi}_{\partial}^{\alpha}\left(x_{1}\right) \pi \bar{\Phi}_{G}^{\beta}\left(x_{2}, x_{2}\right)\right. \\
\left.-\frac{1}{N_{c}^{2}-1} \pi \Phi_{G}^{\alpha}\left(x_{1}, x_{1}\right) \pi \bar{\Phi}_{G}^{\beta}\left(x_{2}, x_{2}\right)\right) \hat{\sigma}_{D Y} .
\end{gathered}
$$

This indicates a breaking of universality for the double gluonic pole contribution. This breaking is consistent with non-factorizability of DY at twist four (actually double twistthree), which would be the level needed to consider similar asymmetries in a collinear treatment that employs gluonic pole matrix elements and where one ought to find the same breaking. It also shows that TMD factorization of DY does not hold for the T-odd parts in the quark-quark correlators. The actual outcome is a strong suppression of the double T-odd contributions and a sign change (-1/8 as compared to 1$)$.

In the same way as for DY, we can analyze higher moments in other processes for which we will consider the example of quark-quark scattering. From the unintegrated result in eq. (4.7) one gets the double-weighted result,

$$
\begin{aligned}
& \left\langle p_{1 T}^{\alpha} p_{2 T}^{\beta} \sigma_{1}\right\rangle \sim \operatorname{Tr}_{c}\left[\widetilde{\Phi}_{\partial}^{\alpha}\left(x_{1}\right) \Gamma_{1}^{*} \Delta\left(z_{1}\right) \Gamma_{1}\right] \operatorname{Tr}_{c}\left[\widetilde{\Phi}_{\partial}^{\beta}\left(x_{2}\right) \Gamma_{2}^{*} \Delta\left(z_{2}\right) \Gamma_{2}\right] \\
& +\operatorname{Tr}_{c}\left[\widetilde{\Phi}_{\partial}^{\alpha}\left(x_{1}\right) \Gamma_{1}^{*} \Delta\left(z_{1}\right) \Gamma_{1}\right] \operatorname{Tr}_{c}\left[\Phi_{8}\left(x_{2}\right) \pi \widetilde{G}^{n \beta}\left[p_{2}\right] \Gamma_{2}^{*} \Delta\left(z_{2}\right) \Gamma_{2}\right] \\
& +\operatorname{Tr}_{c}\left[\Phi_{8}\left(x_{1}\right) \pi \widetilde{G}^{n \alpha}\left[p_{1}\right] \Gamma_{1}^{*} \Delta\left(z_{1}\right) \Gamma_{1}\right] \operatorname{Tr}_{c}\left[\widetilde{\Phi}_{\partial}^{\beta}\left(x_{2}\right) \Gamma_{2}^{*} \Delta\left(z_{2}\right) \Gamma_{2}\right] \\
& +\operatorname{Tr}_{c}\left[\widetilde{\Phi}_{\partial}^{\alpha}\left(x_{1}\right) 2 \pi \widetilde{G}^{n \beta}\left[p_{2}\right] \Gamma_{1}^{*} \Delta\left(z_{1}\right) \Gamma_{1}\right] \operatorname{Tr}_{c}\left[\Phi_{8}\left(x_{2}\right) \Gamma_{2}^{*} \Delta\left(z_{2}\right) \Gamma_{2}\right] \\
& +\operatorname{Tr}_{c}\left[\Phi_{8}\left(x_{1}\right) \Gamma_{1}^{*} \Delta\left(z_{1}\right) \Gamma_{1}\right] \operatorname{Tr}_{c}\left[\widetilde{\Phi}_{\partial}^{\beta}\left(x_{2}\right) 2 \pi \widetilde{G}^{n \alpha}\left[p_{1}\right] \Gamma_{2}^{*} \Delta\left(z_{2}\right) \Gamma_{2}\right] \\
& +\operatorname{Tr}_{c}\left[\Phi_{8}\left(x_{1}\right) \frac{1}{2}\left\{\pi \widetilde{G}^{n \alpha}\left[p_{1}\right], \pi \widetilde{G}^{n \beta}\left[p_{2}\right]\right\} \Gamma_{1}^{*} \Delta\left(z_{1}\right) \Gamma_{1}\right] \operatorname{Tr}_{c}\left[\Phi_{8}\left(x_{2}\right) \Gamma_{2}^{*} \Delta\left(z_{2}\right) \Gamma_{2}\right] \\
& +\operatorname{Tr}_{c}\left[\pi \widetilde{G}^{n \beta}\left[p_{2}\right] \Phi_{8}\left(x_{1}\right) \pi \widetilde{G}^{n \alpha}\left[p_{1}\right] \Gamma_{1}^{*} \Delta\left(z_{1}\right) \Gamma_{1}\right] \operatorname{Tr}_{c}\left[\Phi_{8}\left(x_{2}\right) \Gamma_{2}^{*} \Delta\left(z_{2}\right) \Gamma_{2}\right] \\
& +\operatorname{Tr}_{c}\left[\Phi_{8}\left(x_{1}\right) \Gamma_{1}^{*} \Delta\left(z_{1}\right) \Gamma_{1}\right] \operatorname{Tr}_{c}\left[\Phi_{8}\left(x_{2}\right) \frac{1}{2}\left\{\pi \widetilde{G}^{n \beta}\left[p_{2}\right], \pi \widetilde{G}^{n \alpha}\left[p_{1}\right]\right\} \Gamma_{2}^{*} \Delta\left(z_{2}\right) \Gamma_{2}\right] \\
& +\operatorname{Tr}_{c}\left[\Phi_{8}\left(x_{1}\right) \Gamma_{1}^{*} \Delta\left(z_{1}\right) \Gamma_{1}\right] \operatorname{Tr}_{c}\left[\pi \widetilde{G}^{n \alpha}\left[p_{1}\right] \Phi_{8}\left(x_{2}\right) \pi \widetilde{G}^{n \beta}\left[p_{2}\right] \Gamma_{2}^{*} \Delta\left(z_{2}\right) \Gamma_{2}\right] \\
& +\operatorname{Tr}_{c}\left[\Phi_{8}\left(x_{1}\right) \pi \widetilde{G}^{n \alpha}\left[p_{1}\right] \Gamma_{1}^{*} \Delta\left(z_{1}\right) \Gamma_{1}\right] \operatorname{Tr}_{c}\left[\Phi_{8}\left(x_{2}\right) \pi \widetilde{G}^{n \beta}\left[p_{2}\right] \Gamma_{2}^{*} \Delta\left(z_{2}\right) \Gamma_{2}\right] \\
& +\operatorname{Tr}_{c}\left[\Phi_{8}\left(x_{1}\right) \pi \widetilde{G}^{n \beta}\left[p_{2}\right] \Gamma_{1}^{*} \Delta\left(z_{1}\right) \Gamma_{1}\right] \operatorname{Tr}_{c}\left[\Phi_{8}\left(x_{2}\right) \pi \widetilde{G}^{n \alpha}\left[p_{1}\right] \Gamma_{2}^{*} \Delta\left(z_{2}\right) \Gamma_{2}\right] \\
& +\operatorname{Tr}_{c}\left[\Phi_{8}\left(x_{1}\right) \pi \widetilde{G}^{n \beta}\left[p_{2}\right] \Gamma_{1}^{*} \Delta\left(z_{1}\right) \Gamma_{1}\right] \operatorname{Tr}_{c}\left[\pi \widetilde{G}^{n \alpha}\left[p_{1}\right] \Phi_{8}\left(x_{2}\right) \Gamma_{2}^{*} \Delta\left(z_{2}\right) \Gamma_{2}\right] \\
& +\operatorname{Tr}_{c}\left[\pi \widetilde{G}^{n \beta}\left[p_{2}\right] \Phi_{8}\left(x_{1}\right) \Gamma_{1}^{*} \Delta\left(z_{1}\right) \Gamma_{1}\right] \operatorname{Tr}_{c}\left[\Phi_{8}\left(x_{2}\right) \pi \widetilde{G}^{n \alpha}\left[p_{1}\right] \Gamma_{2}^{*} \Delta\left(z_{2}\right) \Gamma_{2}\right] \\
& +\operatorname{Tr}_{c}\left[\pi \widetilde{G}^{n \beta}\left[p_{2}\right] \Phi_{8}\left(x_{1}\right) \Gamma_{1}^{*} \Delta\left(z_{1}\right) \Gamma_{1}\right] \operatorname{Tr}_{c}\left[\pi \widetilde{G}^{n \alpha}\left[p_{1}\right] \Phi_{8}\left(x_{2}\right) \Gamma_{2}^{*} \Delta\left(z_{2}\right) \Gamma_{2}\right] .(5.33)
\end{aligned}
$$

Several terms are trivially zero after color tracing (four through nine). The others give particular transverse moments, where in particular for the last terms one must evaluate the extra color factor because of the specific color flow. To be specific for terms eleven through fourteen one needs

$$
\frac{\operatorname{Tr}_{c}\left[T^{a} T^{b}\right] \operatorname{Tr}_{c}\left[T^{b} T^{a}\right]}{\operatorname{Tr}_{c}\left[T^{a} T^{a}\right] \operatorname{Tr}_{c}\left[T^{b} T^{b}\right]}=\frac{1}{N_{c}^{2}-1}
$$


as compared to term ten. The result then is

$$
\begin{aligned}
\left\langle p_{1 T}^{\alpha} p_{2 T}^{\beta} \sigma_{1}\right\rangle \sim & \left(\widetilde{\Phi}_{\partial}^{\alpha}\left(x_{1}\right) \widetilde{\Phi}_{\partial}^{\beta}\left(x_{2}\right)+\widetilde{\Phi}_{\partial}^{\alpha}\left(x_{1}\right) \pi \Phi_{G}^{\beta}\left(x_{2}, x_{2}\right)+\pi \Phi_{G}^{\alpha}\left(x_{1}, x_{1}\right) \widetilde{\Phi}_{\partial}^{\beta}\left(x_{2}\right)\right. \\
& \left.+\frac{N_{c}^{2}+3}{N_{c}^{2}-1} \pi \Phi_{G}^{\alpha}\left(x_{1}, x_{1}\right) \pi \Phi_{G}^{\beta}\left(x_{2}, x_{2}\right)\right) \hat{\Sigma}_{1} \Delta\left(z_{1}\right) \Delta\left(z_{2}\right)
\end{aligned}
$$

From the unintegrated result in eq. (4.12) one gets

$$
\begin{aligned}
\left\langle p_{1 T}^{\alpha} p_{2 T}^{\beta} \sigma_{2}\right\rangle \sim & \operatorname{Tr}_{c}\left[\widetilde{\Phi}_{\partial}^{\alpha}\left(x_{1}\right) \Gamma_{1}^{*} \Delta\left(z_{1}\right) \Gamma_{2} \widetilde{\Phi}_{\partial}^{\beta}\left(x_{2}\right) \Gamma_{2}^{*} \Delta\left(z_{2}\right) \Gamma_{1}\right] \\
& +\operatorname{Tr}_{c}\left[\widetilde{\Phi}_{\partial}^{\alpha}\left(x_{1}\right) \Gamma_{1}^{*} \Delta\left(z_{1}\right) \Gamma_{2} \Phi_{8}\left(x_{2}\right) \pi \widetilde{G}^{n \beta}\left[p_{2}\right] \Gamma_{2}^{*} \Delta\left(z_{2}\right) \Gamma_{1}\right] \\
& +\operatorname{Tr}_{c}\left[\Phi_{8}\left(x_{1}\right) \pi \widetilde{G}^{n \alpha}\left[p_{1}\right] \Gamma_{1}^{*} \Delta\left(z_{1}\right) \Gamma_{2} \widetilde{\Phi}_{\partial}^{\beta}\left(x_{2}\right) \Gamma_{2}^{*} \Delta\left(z_{2}\right) \Gamma_{1}\right] \\
& +\operatorname{Tr}_{c}\left[\widetilde{\Phi}_{\partial}^{\alpha}\left(x_{1}\right) 2 \pi \widetilde{G}^{n \beta}\left[p_{2}\right] \Gamma_{1}^{*} \Delta\left(z_{1}\right) \Gamma_{2} \Phi_{8}\left(x_{2}\right) \Gamma_{2}^{*} \Delta\left(z_{2}\right) \Gamma_{1}\right] \\
& +\operatorname{Tr}_{c}\left[\Phi_{8}\left(x_{1}\right) \Gamma_{1}^{*} \Delta\left(z_{1}\right) \Gamma_{2} \widetilde{\Phi}_{\partial}^{\beta}\left(x_{2}\right) 2 \pi \widetilde{G}^{n \alpha}\left[p_{1}\right] \Gamma_{2}^{*} \Delta\left(z_{2}\right) \Gamma_{1}\right] \\
& +\operatorname{Tr}_{c}\left[\Phi_{8}\left(x_{1}\right) \frac{1}{2}\left\{\pi \widetilde{G}^{n \alpha}\left[p_{1}\right], \pi \widetilde{G}^{n \beta}\left[p_{2}\right]\right\} \Gamma_{1}^{*} \Delta\left(z_{1}\right) \Gamma_{2} \Phi_{8}\left(x_{2}\right) \Gamma_{2}^{*} \Delta\left(z_{2}\right) \Gamma_{1}\right] \\
& +\operatorname{Tr}_{c}\left[\pi \widetilde{G}^{n \beta}\left[p_{2}\right] \Phi_{8}\left(x_{1}\right) \pi \widetilde{G}^{n \alpha}\left[p_{1}\right] \Gamma_{1}^{*} \Delta\left(z_{1}\right) \Gamma_{2} \Phi_{8}\left(x_{2}\right) \Gamma_{2}^{*} \Delta\left(z_{2}\right) \Gamma_{1}\right] \\
& +\operatorname{Tr}_{c}\left[\Phi_{8}\left(x_{1}\right) \Gamma_{1}^{*} \Delta\left(z_{1}\right) \Gamma_{2} \Phi_{8}\left(x_{2}\right) \frac{1}{2}\left\{\pi \widetilde{G}^{n \beta}\left[p_{2}\right], \pi \widetilde{G}^{n \alpha}\left[p_{1}\right]\right\} \Gamma_{2}^{*} \Delta\left(z_{2}\right) \Gamma_{1}\right] \\
& +\operatorname{Tr}_{c}\left[\Phi_{8}\left(x_{1}\right) \Gamma_{1}^{*} \Delta\left(z_{1}\right) \Gamma_{2} \pi \widetilde{G}^{n \alpha}\left[p_{1}\right] \Phi_{8}\left(x_{2}\right) \pi \widetilde{G}^{n \beta}\left[p_{2}\right] \Gamma_{2}^{*} \Delta\left(z_{2}\right) \Gamma_{1}\right] \\
& +\operatorname{Tr}_{c}\left[\Phi_{8}\left(x_{1}\right) \pi \widetilde{G}^{n \alpha}\left[p_{1}\right] \Gamma_{1}^{*} \Delta\left(z_{1}\right) \Gamma_{2} \Phi_{8}\left(x_{2}\right) \pi \widetilde{G}^{n \beta}\left[p_{2}\right] \Gamma_{2}^{*} \Delta\left(z_{2}\right) \Gamma_{1}\right] \\
& +\operatorname{Tr}_{c}\left[\Phi_{8}\left(x_{1}\right) \pi \widetilde{G}^{n \beta}\left[p_{2}\right] \Gamma_{1}^{*} \Delta\left(z_{1}\right) \Gamma_{2} \Phi_{8}\left(x_{2}\right) \pi \widetilde{G}^{n \alpha}\left[p_{1}\right] \Gamma_{2}^{*} \Delta\left(z_{2}\right) \Gamma_{1}\right] \\
& +\operatorname{Tr}_{c}\left[\Phi_{8}\left(x_{1}\right) \pi \widetilde{G}^{n \beta}\left[p_{2}\right] \Gamma_{1}^{*} \Delta\left(z_{1}\right) \Gamma_{2} \pi \widetilde{G}^{n \alpha}\left[p_{1}\right] \Phi_{8}\left(x_{2}\right) \Gamma_{2}^{*} \Delta\left(z_{2}\right) \Gamma_{1}\right] \\
& +\operatorname{Tr}_{c}\left[\pi \widetilde{G}^{n \beta}\left[p_{2}\right] \Phi_{8}\left(x_{1}\right) \Gamma_{1}^{*} \Delta\left(z_{1}\right) \Gamma_{2} \Phi_{8}\left(x_{2}\right) \pi \widetilde{G}^{n \alpha}\left[p_{1}\right] \Gamma_{2}^{*} \Delta\left(z_{2}\right) \Gamma_{1}\right] \\
& +\operatorname{Tr}_{c}\left[\pi \widetilde{G}^{n \beta}\left[p_{2}\right] \Phi_{8}\left(x_{1}\right) \Gamma_{1}^{*} \Delta\left(z_{1}\right) \Gamma_{2} \pi \widetilde{G}^{n \alpha}\left[p_{1}\right] \Phi_{8}\left(x_{2}\right) \Gamma_{2}^{*} \Delta\left(z_{2}\right) \Gamma_{1}\right] . \quad(5.55
\end{aligned}
$$

To rewrite everything in terms of standard transverse moments $\Phi_{G}^{\alpha}$ we have to compare the color structure of the terms with two gluonic poles with the standard color structure for $\sigma_{2}$. We need the ratio

$$
\frac{\operatorname{Tr}_{c}\left[T^{a} T^{b} T^{a} T^{b}\right]}{\operatorname{Tr}_{c}\left[T^{a} T^{a} T^{b} T^{b}\right]}=-\frac{1}{N_{c}^{2}-1}
$$

We note that the factor $\operatorname{Tr}_{c}\left[T^{a} T^{a} T^{b} T^{b}\right] / \operatorname{Tr}_{c}\left[T^{a} T^{a}\right] \operatorname{Tr}_{c}\left[T^{b} T^{b}\right]=1 / N_{c}$ is already incorporated in the factors multiplying $\hat{\Sigma}_{1}$ and $\hat{\Sigma}_{2}$. The result is

$$
\begin{aligned}
\left\langle p_{1 T}^{\alpha} p_{2 T}^{\beta} \sigma_{2}\right\rangle \sim & \left(\widetilde{\Phi}_{\partial}^{\alpha}\left(x_{1}\right) \widetilde{\Phi}_{\partial}^{\beta}\left(x_{2}\right)+3 \widetilde{\Phi}_{\partial}^{\alpha}\left(x_{1}\right) \pi \Phi_{G}^{\beta}\left(x_{2}, x_{2}\right)+3 \pi \Phi_{G}^{\alpha}\left(x_{1}, x_{1}\right) \widetilde{\Phi}_{\partial}^{\beta}\left(x_{2}\right)\right. \\
& \left.+\frac{6 N_{c}^{2}-9}{N_{c}^{2}-1} \pi \Phi_{G}^{\alpha}\left(x_{1}, x_{1}\right) \pi \Phi_{G}^{\beta}\left(x_{2}, x_{2}\right)\right) \hat{\Sigma}_{2} \Delta\left(z_{1}\right) \Delta\left(z_{2}\right)
\end{aligned}
$$

Combining the terms we get

$$
\left\langle p_{1 T}^{\alpha} p_{2 T}^{\beta} \sigma\right\rangle \sim \widetilde{\Phi}_{\partial}^{\alpha}\left(x_{1}\right) \widetilde{\Phi}_{\partial}^{\beta}\left(x_{2}\right) \underbrace{\left(\frac{N_{c}^{2}+1}{N_{c}^{2}-1} \hat{\Sigma}_{1}-\frac{2}{N_{c}^{2}-1} \hat{\Sigma}_{2}\right)}_{\hat{\Sigma}} \Delta\left(z_{1}\right) \Delta\left(z_{2}\right)
$$




$$
\begin{aligned}
& +\left(\widetilde{\Phi}_{\partial}^{\alpha}\left(x_{1}\right) \pi \Phi_{G}^{\beta}\left(x_{2}, x_{2}\right)+\pi \Phi_{G}^{\beta}\left(x_{1}, x_{1}\right) \widetilde{\Phi}_{\partial}^{\alpha}\left(x_{2}\right)\right) \underbrace{\left(\frac{N_{c}^{2}+1}{N_{c}^{2}-1} \hat{\Sigma}_{1}-\frac{6}{N_{c}^{2}-1} \hat{\Sigma}_{2}\right)}_{\hat{\Sigma}_{G P}} \Delta\left(z_{1}\right) \Delta\left(z_{2}\right) \\
& +\pi \Phi_{G}^{\alpha}\left(x_{1}, x_{1}\right) \pi \Phi_{G}^{\beta}\left(x_{2}, x_{2}\right)\left(\frac{\left(N_{c}^{2}+1\right)\left(N_{c}^{2}+3\right)}{\left(N_{c}^{2}-1\right)^{2}} \hat{\Sigma}_{1}-\frac{12 N_{c}^{2}-18}{\left(N_{c}^{2}-1\right)^{2}} \hat{\Sigma}_{2}\right) \Delta\left(z_{1}\right) \Delta\left(z_{2}\right),(5.37)
\end{aligned}
$$

which in our example of distinguishable quarks $\left(\hat{\Sigma}_{1}=\hat{\Sigma}_{2}\right)$ gives

$$
\begin{aligned}
\left\langle p_{1 T}^{\alpha} p_{2 T}^{\beta} \sigma\right\rangle \sim & \widetilde{\Phi}_{\partial}^{\alpha}\left(x_{1}\right) \widetilde{\Phi}_{\partial}^{\beta}\left(x_{2}\right) \hat{\Sigma} \Delta\left(z_{1}\right) \Delta\left(z_{2}\right) \\
& +\left(\widetilde{\Phi}_{\partial}^{\alpha}\left(x_{1}\right) \pi \Phi_{G}^{\beta}\left(x_{2}, x_{2}\right)+\pi \Phi_{G}^{\alpha}\left(x_{1}, x_{1}\right) \widetilde{\Phi}_{\partial}^{\beta}\left(x_{2}\right)\right) \frac{N_{c}^{2}-5}{N_{c}^{2}-1} \hat{\Sigma} \Delta\left(z_{1}\right) \Delta\left(z_{2}\right) \\
& +\pi \Phi_{G}^{\alpha}\left(x_{1}, x_{1}\right) \pi \Phi_{G}^{\beta}\left(x_{2}, x_{2}\right) \frac{\left(N_{c}^{4}-8 N_{c}^{2}+21\right)}{\left(N_{c}^{2}-1\right)^{2}} \hat{\Sigma} \Delta\left(z_{1}\right) \Delta\left(z_{2}\right) \\
= & \left(\widetilde{\Phi}_{\partial}^{\alpha}\left(x_{1}\right) \widetilde{\Phi}_{\partial}^{\beta}\left(x_{2}\right) \hat{\Sigma}+\left(\widetilde{\Phi}_{\partial}^{\alpha}\left(x_{1}\right) \pi \Phi_{G}^{\beta}\left(x_{2}, x_{2}\right)+\pi \Phi_{G}^{\alpha}\left(x_{1}, x_{1}\right) \widetilde{\Phi}_{\partial}^{\beta}\left(x_{2}\right)\right) \frac{1}{2} \hat{\Sigma}\right. \\
& \left.+\pi \Phi_{G}^{\alpha}\left(x_{1}, x_{1}\right) \pi \Phi_{G}^{\beta}\left(x_{2}, x_{2}\right) \frac{15}{32} \hat{\Sigma}\right) \Delta\left(z_{1}\right) \Delta\left(z_{2}\right) .
\end{aligned}
$$

The result shows that while the effect of a single gluonic pole is suppressed (factor $1 / 2$ ) as compared to the naive TMD factorization, the effect of the double gluonic pole (double Sivers or double Boer-Mulders) is suppressed less than the naive expectation expected on the basis of single gluonic pole effects that are expected to show up in single spin asymmetries (factor $15 / 32$ versus $1 / 4$ ).

Although these examples for DY and hadron-hadron scattering show that TMD factorization fails, we think that the starting point eq. (3.24) at the end of section 3 appears to be useful, incorporating all leading matrix elements based on a counting of canonical dimensions. From that expression, one can proceed and calculate higher weighted asymmetries.

\section{Conclusions}

In this paper we have used a diagrammatic approach to analyse the leading contributions at tree-level in hard processes in which several hadrons are involved. The diagrammatic approach combines correlators involving hadron states and parton fields with hard partonic amplitudes. The correlators depend on (on-shell) hadron momenta (initial state hadrons $P$, produced hadrons $K_{h}$ ) and the parton momenta ( $p$ and $k$ ). At high energies, momentum fractions $x P$ or $(1 / z) K_{h}$ and transverse momenta can be used, while the components $p \cdot P$ and $k \cdot K_{h}$ are integrated over. At that stage one has correlators that also have a natural link to light-cone wave functions $[42,43]$. In the usual collinear treatment, the transverse momenta are also integrated over, or one studies the limit in which these momenta become large, which just is a collinear treatment involving a partonic process with one or more additional partons. Using TMD correlators one aims at incorporating all features related to (soft) transverse momenta, including in the parametrization of TMDs the possibility of $T$-odd correlators, not forbidden by any symmetry. These incorporate the effects of 
initial and final state interactions [44] and they can explain the existence of single spin asymmetries at high energies.

The basic tree-level result was presented in section 3. It includes all Wilson lines originating from collinear gluons in all of the correlators. These contributions are sufficient to study the collinear correlators (integrating over transverse components of the parton momenta) and give rise to straight light-like gauge links. For TMD correlators, one has to include transverse gauge connections, but after accounting for the collinear gluons, this all works out nice and produces the transverse pieces at the right place $[8,9]$. One obtains a color gauge-invariant expression (eq. (3.24)), which is fully entangled.

In order to disentangle color, one needs to consider for each diagrammatic contribution in the hard amplitude, also its color-flow possibilities. This does not resolve the entanglement in general. Also, integrating over transverse momenta of final state partons, e.g. when one considers jet production, does not resolve the problem. Then one remains with an entangled situation if the initial state involves more than one hadron, as in the case of hadron-hadron scattering. This was considered as an explicit example of factorization breaking in ref. [37]. The full entangled tree-level result for this situation is given in eq. (4.7). Even in cases in which one just has a single color flow such as in the electroweak processes of leptoproduction, electron-positron annihilation or the Drell-Yan process, one gets TMD factorization with correlators having specific process-dependent gauge links running via \pm light-cone infinity. For these cases one then has a starting point with universal correlators and one can study the full QCD factorization. There are several examples of similarly simple processes, such as two-photon production in Drell-Yan, which even for gluons has a simple color flow [45].

The main aim of this paper was to show what happens in the special case of an 1PU process, in which only the transverse momentum in one specific hadron is considered. In that situation, one can resolve the entanglement (at least at tree-level). What remains is a color gauge-invariant expression (eq. (4.15)) with a process-dependent gauge link. All-butone collinear processes may seem hard to realize at first sight, since the parton transverse momentum is an integration variable, rather than an observable. Nevertheless, symmetry considerations in combination with polarization, in particular transverse polarization of target hadrons, may help to create the conditions for an 1PU treatment. Furthermore one has in leptoproduction processes a whole class of such processes. Consideration of TMD correlators is possible for quark as well as gluon correlators. A recent example of the latter situation was pointed out in ref. [46] which considered heavy quark production in the Drell-Yan process.

The simplifications and steps towards a TMD factorizable result become more transparant if one constructs transverse moments. This analysis has been given in section 5 for single and double weighted asymmetries. Single weighted asymmetries can use the results of the $1 \mathrm{PU}$ situation and lead to the results where $T$-even transverse moments are convoluted with the standard partonic cross section and $T$-odd transverse moments are convoluted with a different (but color gauge-invariant) combination of squared amplitudes, the so-called gluonic pole cross sections. The $T$-odd transverse moments are weighted TMD correlators, involving $p_{T}^{2}$-weighted $T$-odd distribution functions like the Sivers or 
Boer-Mulders distribution functions of which the operator structure is a gluonic pole matrix element, i.e. a quark-quark-gluon matrix element at vanishing gluon momentum.

While the single weighted asymmetries exhibit factorization at the TMD-level (with process-dependence through the link in the TMD) and at the collinear level involve gluonic pole cross sections, rather than the standard partonic cross sections, the explicit evaluation of the double weighted asymmetry shows that such factorizations are not generally applicable. Additional color factors come in at this level, which provide besides double weighted TMDs for hadron 1 and hadron 2, products of single weighted functions. The factors multiplying the final result for the cross terms, however, is not related to the factors appearing in the single weighted results. It does involve products of the $T$-even and $T$-odd transverse moments, but each of these terms with their own factors.

The analysis in terms of transverse moments, however, also provides us with an important result for correlators belonging to final state hadrons. In analogy to the analysis of distribution correlators, the transverse moments for fragmentation correlators involve a part $\widetilde{\Delta}_{\partial}^{\alpha}$ with a $T$-even combination of operators and a part $\Delta_{G}^{\alpha}$ with a $T$-odd combination of operators, the latter involving an integration over transverse gluon fields corresponding to vanishing gluon momentum. Such gluonic pole matrix elements (and also multi-gluonic pole matrix elements appearing in higher transverse moments) vanish for fragmentation. This has been shown in models as well using spectral analysis of final states [27, 28] or studies of analytic properties [29] based on the field theoretical correspondence to amplitudes mentioned at the end of section 2. Since the color structure of the remaining $\widetilde{\Delta}_{\partial}^{\alpha}$ part is simple, there are no complications to absorb the appropriate gluons into gauge links. This implies that the result of eq. (4.7) also applies for unintegrated situations, where the dependence on transverse momenta in the final state is kept. Thus we can use in eq. (4.7) TMD correlators $\Delta\left(z, k_{T}\right)$ rather than the collinear correlators $\Delta(z)$. Instead of eq. (3.24) one has a tree-level result of the form

$$
\begin{aligned}
d \sigma \sim & \operatorname{Tr}_{c}\left[U_{-}^{\dagger}\left[p_{2}\right] \Phi\left(x_{1}, p_{1 T}\right) U_{-}\left[p_{2}\right] W_{+}^{\dagger}\left[p_{1}, p_{2}\right]\right] \operatorname{Tr}_{c}\left[U_{-}^{\dagger}\left[p_{1}\right] \Phi\left(x_{2}, p_{2 T}\right) U_{-}\left[p_{1}\right] W_{+}^{\dagger}\left[p_{1}, p_{2}\right]\right] \\
& \times \Gamma_{1}^{*} \Delta\left(z_{1}, k_{1 T}\right) \Gamma_{1} \Gamma_{2}^{*} \Delta\left(z_{2}, k_{2 T}\right) \Gamma_{2}
\end{aligned}
$$

with entanglement only involving the initial state TMD correlators, including dependence on color flow in initial and final state. In spite of having a $T$-even operator structure, the universal TMD fragmentation correlators $\Delta\left(z, k_{T}\right)$ or its transverse moment $\widetilde{\Delta}_{\partial}^{\alpha}(z)$ allows for $T$-odd fragmentation functions in its parametrization such as the Collins function because the non-plane-wave nature of the states $\left|K_{h}, K_{X}\right\rangle$ in the matrix elements of eq. (2.5) prevents using time-reversal symmetry constraints.

The formalism in this paper allows a rich phenomenology, including many results on single transverse momentum weighted cross sections that have already been obtained. It can be used for quark as well as gluon TMDs, although the latter involve a number of additional complications. However, we do want to emphasize once more that our results are strictly tree-level, which does provide insight into the universality of TMDs and which is a necessary condition to study factorization issues, but it does not provide proof of factorization in QCD. For a discussion of those aspects we refer to ref. [17], to ref. [16] on 
subtleties with gauge link structures at higher orders and to ref. [18] for attempts to bridge the gap between the phenomenology and the more formal definitions.

\section{Acknowledgments}

We would like to acknowledge early stage contributions of Christiaan Mantz and discussions with Daniel Boer, Mert Aybat, Ted Rogers. Also acknowledged are discussions with JanWei Qiu, John Collins, Yuji Koike and several others at the stimulating Workshop on Opportunities for Drell-Yan at RHIC at Brookhaven National Laboratory (11-13 May 2011). This work is part of research programs of the foundation for Fundamental Research of Matter (FOM) and the National Organization for Scientific Research (NWO) as well as the FP 7 EU-program Hadron Physics (No. 227431). All figures were made using Jaxodraw [47, 48].

\section{A Collinear gauge links}

In this appendix we incorporate the collinear gluons, i.e. the first term in the Sudakov expansion of $A^{\mu}(p)$ given in eq. (3.4). This will produce the parts of the gauge link along light-like directions conjugate to the momentum $P$ and in the collinear situation (when all transverse momenta are integrated over) it produces the gauge link as given in eq. (2.17) in the light-cone correlators.

Looking at the Fourier transformed fields with collinear Wilson lines starting at $\pm \infty$,

we consider the Fourier transform of the field $U_{[ \pm \infty, \xi]}^{[n]} \psi(\xi)$. For the field with Wilson line starting at minus infinity we get,

$$
\underline{L}_{-}^{[n]} \psi(p)=\int d^{4} \xi \exp (i p \cdot \xi) \mathscr{P} \exp \left(-i g \int_{-\infty}^{\xi \cdot P} d(\eta \cdot P) n \cdot A(\eta)\right) \psi(x) .
$$

Looking only at the relevant component $p^{+}=p \cdot n$ one gets,

$$
\begin{aligned}
\underbrace{L_{-}^{[n]} \psi}_{-}(p)=\sum_{N=0}^{\infty}(-i)^{N} \int_{-\infty}^{\infty} d \xi^{-} & \int_{-\infty}^{\xi} d \eta_{N}^{-} \int_{\eta_{N}^{-}}^{\xi} d \eta_{N-1}^{-} \ldots \\
& \times \int_{\eta_{2}^{-}}^{\xi} d \eta_{1}^{-} A^{+}\left(\eta_{N}^{-}\right) \ldots A^{+}\left(\eta_{1}^{-}\right) \psi(\xi) e^{i p^{+} \xi^{-}}
\end{aligned}
$$

where the arguments run between $-\infty<\eta_{N}^{-}<\eta_{N-1}^{-}<\ldots<\eta_{1}^{-}<\xi^{-}$, implemented through $\theta$ functions $\theta\left(\eta_{N-1}^{-}-\eta_{N}^{-}\right) \ldots \theta\left(\eta_{1}^{-}-\eta_{2}^{-}\right) \theta\left(\xi^{-}-\eta_{1}^{-}\right)$, which can be rewritten as momentum-space integrations,

$$
\begin{aligned}
\underline{L}_{-}^{[n]} \psi\left(p^{+}\right)= & \sum_{N=0}^{\infty}(-i)^{N} \int_{-\infty}^{\infty} d \xi^{-} \int_{-\infty}^{\infty} d \eta_{N}^{-} \int_{-\infty}^{\infty} d \eta_{N-1}^{-} \ldots \int_{-\infty}^{\infty} d \eta_{1}^{-} \\
& \times \int \frac{d p_{N}^{+}}{-2 \pi i} \ldots \int \frac{d p_{1}^{+}}{-2 \pi i} \frac{e^{-i p_{N}^{+}\left(\eta_{N-1}^{-}-\eta_{N}^{-}\right)}}{p_{N}^{+}+i \epsilon} \ldots \frac{e^{-i p_{1}^{+}\left(\xi^{-}-\eta_{1}^{-}\right)}}{p_{1}^{+}+i \epsilon} \\
& \times A^{+}\left(\eta_{N}^{-}\right) \ldots A^{+}\left(\eta_{1}^{-}\right) \psi\left(\xi^{-}\right) e^{i p^{+} \xi^{-}} .
\end{aligned}
$$


Including again the dependence on other momenta, we find

$$
\begin{gathered}
\underset{\underline{-}^{[n]} \psi(p)=}{ } \sum_{N=0}^{\infty} \int \frac{d^{4} p_{N}}{(2 \pi)^{4}} \ldots \int \frac{d^{4} p_{1}}{(2 \pi)^{4}} \frac{A^{n}\left(p_{N}\right)}{\left(x_{N}+i \epsilon\right)} \frac{A^{n}\left(p_{N-1}-p_{N}\right)}{\left(x_{N-1}+i \epsilon\right)} \ldots \frac{A^{n}\left(p_{1}-p_{2}\right)}{\left(x_{1}+i \epsilon\right)} \psi\left(p-p_{1}\right) \\
=\sum_{N=0}^{\infty} \int \frac{d^{4} p_{1}}{(2 \pi)^{4}} \ldots \int \frac{d^{4} p_{N}}{(2 \pi)^{4}} \frac{A^{n}\left(p_{1}\right)}{\left(x_{1}+i \epsilon\right)} \frac{A^{n}\left(p_{2}\right)}{\left(x_{1}+x_{2}+i \epsilon\right)} \ldots \\
\times \frac{A^{n}\left(p_{N}\right)}{\left(x_{1}+\cdots+x_{N}+i \epsilon\right)} \psi\left(p-\sum_{i=1}^{N} p_{i}\right),
\end{gathered}
$$

where $x_{i}=p_{i} \cdot n$. We thus find for the gauge link

$$
U_{ \pm}^{[n]} \psi(p)=\sum_{M=0}^{\infty} U_{ \pm}^{[n](M)} \psi(p),
$$

where $U_{ \pm}^{[n](0)}=1$. The gauge link and the terms in its expansion not only have a particular structure in coordinate or momentum space, but they also have a charge structure. In particular for applications in non-abelian gauge theories one has matrix-valued fields $A^{\mu}=A^{\mu a} T^{a}$.

The gauge link can be written in a nicer symmetric form for the correlators. Commutators $\left[A^{n}\left(p_{1}\right), A^{n}\left(p_{2}\right)\right]$ don't matter here, neither in color space, where they are 'contained' in other matrix elements with less gluons, nor in Hilbert space, where one has commuting fields, which for fields with the light-cone index $n$ imply vanishing commutators. We can then use relations

$$
\begin{gathered}
\frac{1}{\left(x_{1}+x_{2}+i \epsilon\right)}\left[\frac{1}{\left(x_{1}+i \epsilon\right)}+\frac{1}{\left(x_{2}+i \epsilon\right)}\right]=\frac{1}{\left(x_{1}+i \epsilon\right)\left(x_{2}+i \epsilon\right)}, \\
\frac{1}{\left(x_{1}+x_{2}+x_{3}+i \epsilon\right)} \underbrace{\left[\frac{1}{\left(x_{1}+x_{2}+i \epsilon\right)\left(x_{1}+i \epsilon\right)}+\cdots\right]}_{6 \text { permutations }} \\
=\frac{1}{\left(x_{1}+x_{2}+x_{3}+i \epsilon\right)} \underbrace{\left[\frac{1}{\left(x_{1}+i \epsilon\right)\left(x_{2}+i \epsilon\right)}+\cdots\right]}_{3 \text { permutations }} \\
=\frac{1}{\left(x_{1}+i \epsilon\right)\left(x_{2}+i \epsilon\right)\left(x_{3}+i \epsilon\right)},
\end{gathered}
$$

and its generalization to more terms, to symmetrize the result. This gives

$$
\begin{aligned}
\underline{L}_{-}^{[n]} \psi(p) & =\int d^{4} \xi \exp (i p \cdot \xi) \mathscr{P} \exp \left(-i g \int_{-\infty}^{\xi \cdot P} d(\eta \cdot P) n \cdot A(\eta)\right) \psi(x) \\
& =\sum_{N=0}^{\infty} \frac{1}{N !} \int \frac{d^{4} p_{1}}{(2 \pi)^{4}} \ldots \int \frac{d^{4} p_{N}}{(2 \pi)^{4}} \frac{A^{n}\left(p_{1}\right) A^{n}\left(p_{2}\right) \ldots A^{n}\left(p_{N}\right)}{\left(x_{1}+i \epsilon\right)\left(x_{2}+i \epsilon\right) \ldots\left(x_{N}+i \epsilon\right)} \psi\left(p-\sum_{i=1}^{N} p_{i}\right) .
\end{aligned}
$$

From this expression one sees that the term $U_{ \pm}^{[n](M)}$ is the consecutive action of $M$ simple (commuting) $U_{ \pm}^{[n](1)}$-connections 


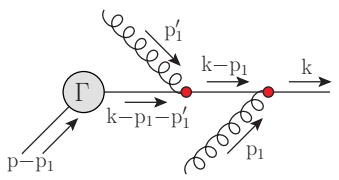

(a)

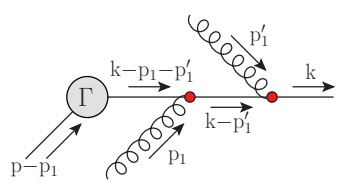

(b)

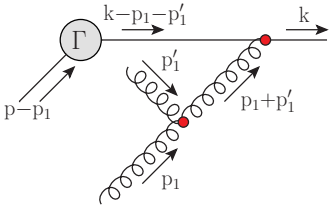

(c)

Figure 9. The gluon insertions on an outgoing quark line coming from two different soft pieces, one from $\Phi(p)$ and one from $\Phi\left(p^{\prime}\right)$, respectively.

For a link along $n$ coming from $+\infty$ one has

$$
\begin{aligned}
& \underbrace{U_{+}^{[n]}}_{+} \psi(p)=\int d^{4} \xi \exp (i p \cdot \xi) \mathscr{P} \exp \left(-i g \int_{\infty}^{\xi \cdot P} d(\eta \cdot P) n \cdot A(\eta)\right) \psi(x) \\
&=\sum_{N=0}^{\infty} \int \frac{d^{4} p_{N}}{(2 \pi)^{4}} \ldots \int \frac{d^{4} p_{1}}{(2 \pi)^{4}} \frac{A^{n}\left(p_{N}\right)}{\left(-x_{N}+i \epsilon\right)} \frac{A^{n}\left(p_{N-1}-p_{N}\right)}{\left(-x_{N-1}+i \epsilon\right)} \ldots \frac{A^{n}\left(p_{1}-p_{2}\right)}{\left(-x_{1}+i \epsilon\right)} \psi\left(p-p_{1}\right) \\
&=\sum_{N=0}^{\infty} \int \frac{d^{4} p_{1}}{(2 \pi)^{4}} \ldots \int \frac{d^{4} p_{N}}{(2 \pi)^{4}} \frac{A^{n}\left(p_{1}\right)}{\left(-x_{1}+i \epsilon\right)} \ldots \frac{A^{n}\left(p_{N}\right)}{\left(-x_{1}-\cdots-x_{N}+i \epsilon\right)} \psi\left(p-\sum_{i=1}^{N} p_{i}\right) \\
&=\sum_{N=0}^{\infty} \frac{1}{N !} \int \frac{d^{4} p_{1}}{(2 \pi)^{4}} \ldots \int \frac{d^{4} p_{N}}{(2 \pi)^{4}} \frac{A^{n}\left(p_{1}\right) A^{n}\left(p_{2}\right) \ldots A^{n}\left(p_{N}\right)}{\left(-x_{1}+i \epsilon\right)\left(-x_{2}+i \epsilon\right) \ldots\left(-x_{N}+i \epsilon\right)} \\
& \times \psi\left(p-\sum_{i=1}^{N} p_{i}\right) .
\end{aligned}
$$

The notation used in equations with many fields and links will be $U_{+}^{[n]} \psi(p)=U_{+}^{[n]}[p] \psi(p)$.

\section{B Intertwined gauge connections}

We consider the situation of gluon insertions on an outgoing quark line coming from two different soft pieces, one from $\Phi(p)$ and one from $\Phi\left(p^{\prime}\right)$, respectively. There are three leading contributions of $A^{k}\left(p_{1}\right) p_{1}^{\mu}$ and $A^{k}\left(p_{1}^{\prime}\right) p_{1}^{\prime \mu}$ gluon components, corresponding to the diagrams in figure 9 , one of them involving a triple gluon vertex. The combined result of the diagrams actually nicely adds up to

$$
A_{11}=\left[\bar{\psi}(k) U_{+}^{[k](11)}\left[p, p^{\prime}\right] \Gamma \psi(p)\right] \ldots \psi\left(p^{\prime}\right)=\frac{1}{2}\left[\bar{\psi}(k)\left\{U_{+}^{[k](1)}\left[p^{\prime}\right], U_{+}^{[k](1)}[p]\right\} \Gamma \psi(p)\right] \ldots \psi\left(p^{\prime}\right),
$$

which is a gauge connection which is the (color) symmetrized product of simple connections.

In order to illustrate the recursive procedure, it is instructive to give the result for the situation of insertions on an outgoing quark line for three gluons coming from two different soft pieces, two gluons coming from $\Phi(p)$ and one coming from $\Phi\left(p^{\prime}\right)$, respectively. This is shown in figure 10 and involves three- and four-gluon couplings. The result becomes

$$
A_{21}=\left[\bar{\psi}(k) U_{+}^{[k](21)}\left[p, p^{\prime}\right] \Gamma \ldots \psi(p)\right] \ldots \psi\left(p^{\prime}\right)
$$




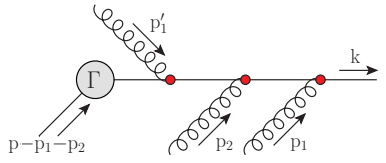

(a)

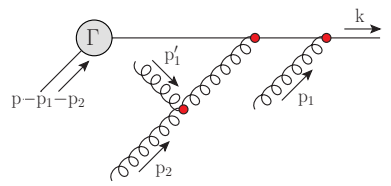

(d)

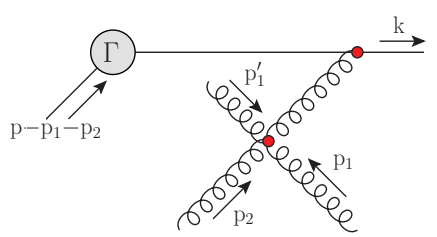

$(\mathrm{g})$

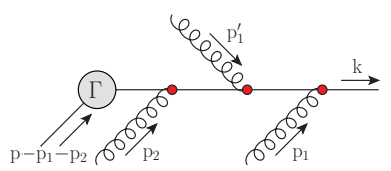

(b)

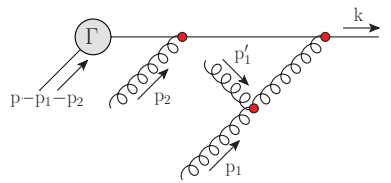

(e)

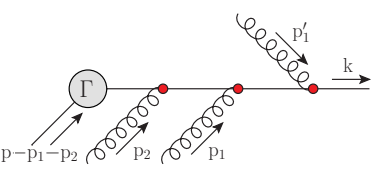

(c)

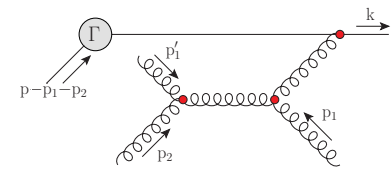

(f)

Figure 10. The gluon insertions on an outgoing quark line for three gluons coming from two different soft pieces, two gluons coming from $\Phi(p)$ and one coming from $\Phi\left(p^{\prime}\right)$, respectively. Note the absence of a three-gluon vertex with gluon legs with momenta $p_{1}$ and $p_{2}$ coupling to the same soft part $\Phi(p)$. Note the absence of a three-gluon vertex with gluon legs with momenta $p_{1}$ and $p_{2}$ coupling to the same soft part $\Phi(p)$. It is actually already included in the soft part with one gluon leg less.

$$
\begin{gathered}
=\left[\overline { \psi } ( k ) \left(\frac{1}{4} U_{+}^{[k](2)}[p] U_{+}^{[k](1)}\left[p^{\prime}\right]+\frac{1}{4} U_{+}^{[k](1)}[p] U_{+}^{[k](1)}\left[p^{\prime}\right] U_{+}^{[k](1)}[p]\right.\right. \\
\left.\left.+\frac{1}{4} U_{+}^{[k](1)}\left[p^{\prime}\right] U_{+}^{[k](2)}[p]\right) \Gamma \ldots \psi(p)\right] \ldots \psi\left(p^{\prime}\right) \\
=\left[\overline { \psi } ( k ) \left(\frac{1}{8} U_{+}^{[k](1)}[p] U_{+}^{[k](1)}[p] U_{+}^{[k](1)}\left[p^{\prime}\right]+\frac{1}{4} U_{+}^{[k](1)}[p] U_{+}^{[k](1)}\left[p^{\prime}\right] U_{+}^{[k](1)}[p]\right.\right. \\
\left.\left.+\frac{1}{8} U_{+}^{[k](1)}\left[p^{\prime}\right] U_{+}^{[k](1)}[p] U_{+}^{[k](1)}[p]\right) \Gamma \ldots \psi(p)\right] \ldots \psi\left(p^{\prime}\right) .
\end{gathered}
$$

For a general $A_{i j k}$-gluon term, with $i-, j$ - and $k$ gluons from three (or more) different correlators, one finds an expression where the $A$-fields from these different correlators are a color symmetrized product of $U^{[k](1)}$ factors containing commuting gauge fields. Thus if one, as is the case in eq. (3.11), looks at the result from insertions on leg $k_{2}$ coming from correlators $\Phi\left(p_{1}\right), \Phi\left(p_{2}\right)$ and $\Delta\left(k_{1}\right)$ it can be broken apart in a symmetrized product of simple gauge links,

$$
U_{+}^{\left[k_{2}\right]}\left[p_{1}, p_{2}, k_{1}\right]=\mathcal{S}\left\{U_{+}^{\left[k_{2}\right]}\left[p_{1}\right] U_{+}^{\left[k_{2}\right]}\left[p_{2}\right] U_{+}^{\left[k_{2}\right]}\left[k_{1}\right]\right\},
$$

of which the ordering is irrelevant.

Open Access. This article is distributed under the terms of the Creative Commons Attribution Noncommercial License which permits any noncommercial use, distribution, and reproduction in any medium, provided the original author(s) and source are credited. 


\section{References}

[1] J.C. Collins and D.E. Soper, Parton distribution and decay functions, Nucl. Phys. B 194 (1982) 445 [SPIRES].

[2] R.L. Jaffe and X.-D. Ji, Chiral odd parton distributions and polarized Drell-Yan, Phys. Rev. Lett. 67 (1991) 552 [SPIRES].

[3] A.V. Efremov and A.V. Radyushkin, Field theoretic treatment of high momentum transfer processes. 3. Gauge theories, Theor. Math. Phys. 44 (1981) 774 [Teor. Mat. Fiz. 44 (1980) 327] [SPIRES].

[4] J.C. Collins, Leading-twist single-transverse-spin asymmetries: Drell-Yan and deep-inelastic scattering, Phys. Lett. B 536 (2002) 43 [hep-ph/0204004] [SPIRES].

[5] J.P. Ralston and D.E. Soper, Production of dimuons from high-energy polarized proton proton collisions, Nucl. Phys. B 152 (1979) 109 [SPIRES].

[6] P.J. Mulders and R.D. Tangerman, The complete tree-level result up to order $1 / Q$ for polarized deep-inelastic leptoproduction, Nucl. Phys. B 461 (1996) 197 [Erratum ibid. B 484 (1997) 538] [hep-ph/9510301] [SPIRES].

[7] D. Boer and P.J. Mulders, Time-reversal odd distribution functions in leptoproduction, Phys. Rev. D 57 (1998) 5780 [hep-ph/9711485] [SPIRES].

[8] A.V. Belitsky, X. Ji and F. Yuan, Final state interactions and gauge invariant parton distributions, Nucl. Phys. B 656 (2003) 165 [hep-ph/0208038] [SPIRES].

[9] D. Boer, P.J. Mulders and F. Pijlman, Universality of T-odd effects in single spin and azimuthal asymmetries, Nucl. Phys. B 667 (2003) 201 [hep-ph/0303034] [SPIRES].

[10] C.J. Bomhof, P.J. Mulders and F. Pijlman, Gauge link structure in quark quark correlators in hard processes, Phys. Lett. B 596 (2004) 277 [hep-ph/0406099] [SPIRES].

[11] C.J. Bomhof, P.J. Mulders and F. Pijlman, The construction of gauge-links in arbitrary hard processes, Eur. Phys. J. C 47 (2006) 147 [hep-ph/0601171] [SPIRES].

[12] C.J. Bomhof and P.J. Mulders, Non-universality of transverse momentum dependent parton distribution functions, Nucl. Phys. B 795 (2008) 409 [arXiv:0709.1390] [SPIRES].

[13] J.-H. Gao, Derivation of the gauge link in light cone gauge, Phys. Rev. D 82 (2010) 014018 [arXiv: 1005.4305] [SPIRES].

[14] A. Bacchetta et al., Semi-inclusive deep inelastic scattering at small transverse momentum, JHEP 02 (2007) 093 [hep-ph/0611265] [SPIRES].

[15] V. Barone, F. Bradamante and A. Martin, Transverse-spin and transverse-momentum effects in high-energy processes, Prog. Part. Nucl. Phys. 65 (2010) 267 [arXiv:1011.0909] [SPIRES].

[16] I.O. Cherednikov, A.I. Karanikas and N.G. Stefanis, Wilson lines in transverse-momentum dependent parton distribution functions with spin degrees of freedom, Nucl. Phys. B 840 (2010) 379 [arXiv:1004.3697] [SPIRES].

[17] J.C. Collins, Foundations of perturbative QCD, Cambridge University Press, Cambridge U.K. (2011).

[18] S.M. Aybat and T.C. Rogers, TMD parton distribution and fragmentation functions with QCD evolution, Phys. Rev. D 83 (2011) 114042 [arXiv:1101.5057] [SPIRES]. 
[19] A.V. Efremov and O.V. Teryaev, On spin effects in quantum chromodynamics, Sov. J. Nucl. Phys. 36 (1982) 140 [Yad. Fiz. 36 (1982) 242] [SPIRES].

[20] A.V. Efremov and O.V. Teryaev, QCD asymmetry and polarized hadron structure functions, Phys. Lett. B 150 (1985) 383 [SPIRES].

[21] J.-W. Qiu and G.F. Sterman, Single transverse spin asymmetries, Phys. Rev. Lett. 67 (1991) 2264 [SPIRES].

[22] J.-W. Qiu and G.F. Sterman, Single transverse spin asymmetries in direct photon production, Nucl. Phys. B 378 (1992) 52 [SPIRES].

[23] J.-W. Qiu and G.F. Sterman, Single transverse-spin asymmetries in hadronic pion production, Phys. Rev. D 59 (1999) 014004 [hep-ph/9806356] [SPIRES].

[24] Y. Kanazawa and Y. Koike, Chiral-odd contribution to single-transverse spin asymmetry in hadronic pion production, Phys. Lett. B 478 (2000) 121 [hep-ph/0001021] [SPIRES].

[25] H. Eguchi, Y. Koike and K. Tanaka, Twist-three formalism for single transverse spin asymmetry reexamined: semi-inclusive deep inelastic scattering, Nucl. Phys. B 763 (2007) 198 [hep-ph/0610314] [SPIRES].

[26] Y. Koike and K. Tanaka, Master formula for twist-three soft-gluon-pole mechanism to single transverse-spin asymmetry, Phys. Lett. B 646 (2007) 232 [Erratum ibid. B 668 (2008) 458] [hep-ph/0612117] [SPIRES].

[27] L.P. Gamberg, A. Mukherjee and P.J. Mulders, Spectral analysis of gluonic pole matrix elements for fragmentation, Phys. Rev. D 77 (2008) 114026 [arXiv:0803.2632] [SPIRES].

[28] S. Meissner and A. Metz, Partonic pole matrix elements for fragmentation, Phys. Rev. Lett. 102 (2009) 172003 [arXiv:0812.3783] [SPIRES].

[29] L.P. Gamberg, A. Mukherjee and P.J. Mulders, A model independent analysis of gluonic pole matrix elements and universality of TMD fragmentation functions,

Phys. Rev. D 83 (2011) 071503 [arXiv:1010.4556] [SPIRES].

[30] A. Bacchetta, D. Boer, M. Diehl and P.J. Mulders, Matches and mismatches in the descriptions of semi-inclusive processes at low and high transverse momentum, JHEP 08 (2008) 023 [arXiv: 0803.0227] [SPIRES].

[31] A.A. Henneman, D. Boer and P.J. Mulders, Evolution of transverse momentum dependent distribution and fragmentation functions, Nucl. Phys. B 620 (2002) 331 [hep-ph/0104271] [SPIRES].

[32] R.L. Jaffe, Parton distribution functions for twist four, Nucl. Phys. B 229 (1983) 205 [SPIRES].

[33] M. Diehl and T. Gousset, Time ordering in off-diagonal parton distributions, Phys. Lett. B 428 (1998) 359 [hep-ph/9801233] [SPIRES].

[34] P.V. Landshoff and J.C. Polkinghorne, Models for hadronic and leptonic processes at high-energy, Phys. Rept. 5 (1972) 1 [SPIRES].

[35] D. Boer and P.J. Mulders, Color gauge invariance in the Drell-Yan process, Nucl. Phys. B 569 (2000) 505 [hep-ph/9906223] [SPIRES].

[36] J.C. Collins, D.E. Soper and G.F. Sterman, Does the Drell-Yan cross-section factorize?, Phys. Lett. B 109 (1982) 388 [SPIRES]. 
[37] T.C. Rogers and P.J. Mulders, No generalized TMD-factorization in the hadro-production of high transverse momentum hadrons, Phys. Rev. D 81 (2010) 094006 [arXiv:1001.2977] [SPIRES].

[38] A. Bacchetta, C.J. Bomhof, P.J. Mulders and F. Pijlman, Single spin asymmetries in hadron hadron collisions, Phys. Rev. D 72 (2005) 034030 [hep-ph/0505268] [SPIRES].

[39] C.J. Bomhof and P.J. Mulders, Gluonic pole cross sections and single spin asymmetries in hadron hadron scattering, JHEP 02 (2007) 029 [hep-ph/0609206] [SPIRES].

[40] H. Beppu, Y. Koike, K. Tanaka and S. Yoshida, Contribution of twist-three multi-gluon correlation functions to single spin asymmetry in semi-inclusive deep inelastic scattering, Phys. Rev. D 82 (2010) 054005 [arXiv: 1007.2034] [SPIRES].

[41] H. Beppu, Y. Koike, K. Tanaka and S. Yoshida, On the contribution of twist-three multi-gluon correlation functions to single transverse-spin asymmetry in SIDIS, J. Phys. Conf. Ser. 295 (2011) 012082 [arXiv: 1012.2220] [SPIRES].

[42] B. Pasquini, S. Boffi, A.V. Efremov and P. Schweitzer, Transverse momentum dependent parton distributions and azimuthal asymmetries in light-cone quark models, arXiv:0912.1761 [SPIRES].

[43] V.M. Braun, T. Lautenschlager, A.N. Manashov and B. Pirnay, Higher twist parton distributions from light-cone wave functions, Phys. Rev. D 83 (2011) 094023 [arXiv:1103.1269] [SPIRES].

[44] S.J. Brodsky, D.S. Hwang and I. Schmidt, Final-state interactions and single-spin asymmetries in semi-inclusive deep inelastic scattering, Phys. Lett. B 530 (2002) 99 [hep-ph/0201296] [SPIRES].

[45] J. Qiu, M. Schlegel and W. Vogelsang, Probing gluonic spin-orbit correlations in photon pair production, arXiv: 1103.3861 [SPIRES].

[46] D. Boer, S.J. Brodsky, P.J. Mulders and C. Pisano, Direct probes of linearly polarized gluons inside unpolarized hadrons, Phys. Rev. Lett. 106 (2011) 132001 [arXiv:1011.4225] [SPIRES].

[47] D. Binosi and L. Theussl, JaxoDraw: a graphical user interface for drawing Feynman diagrams, Comput. Phys. Commun. 161 (2004) 76 [hep-ph/0309015] [SPIRES].

[48] D. Binosi, J. Collins, C. Kaufhold and L. Theussl, JaxoDraw: a graphical user interface for drawing Feynman diagrams. Version 2.0 release notes,

Comput. Phys. Commun. 180 (2009) 1709 [arXiv:0811.4113] [SPIRES]. 